\title{
Rapid magma ascent and formation of the Águas Belas-Canindé granitic batholith, NE Brazil: evidence of epidote dissolution and thermobarometry
}

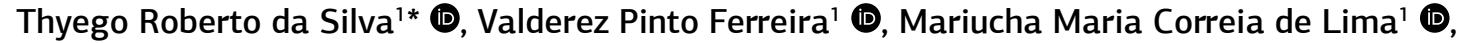 \\ Alcides Nóbrega Sial' (D)
}

\begin{abstract}
Mineral chemistry and intensive parameter estimates for the Major Isidoro (626 Ma) and Monteirópolis (627 Ma) magmatic epidote-bearing granitic plutons, emplaced along the Jacaré dos Homens transpressional shear zone (JHSZ), Borborema Province, are focused in this study. These plutons consist of medium-to-coarse grained equigranular to porphyritic tonalite to granite that show abundant dioritic enclaves. These granites contain biotite (Fe\# 0.44 to 0.55 ), Fe-edenite (Major Isidoro), hastingsite (Monteirópolis), titanite, and epidote that often show allanite core as key mafic mineral phases. Pistacite molecular content in epidote is in the interval of 27 to 31 mol\%, presenting $\mathrm{TiO}_{2}<0.30 \%$, typical for magmatic epidote. Estimated intensive parameters reveal crystallization at $6.5 \pm 1$ (Major Isidoro) and $4.7 \pm 0.6$ $\mathrm{kbar}$ (Monteirópolis), temperatures from $\sim 940^{\circ} \mathrm{C}$ (near-liquidus) to $675 \pm 35^{\circ} \mathrm{C}$ (near-solidus) and oxidizing conditions. Partial corrosion of epidote took place during 15.6 to 32 (Major Isidoro) and 27-49 years (Monteirópolis), corresponding to rather high magma ascension rates of 365 to 750 and 395 to 635 m.years ${ }^{-1}$, respectively. The JHSZ likely favored upward magma transport at the Sergipano and Pernambuco-Alagoas domains boundary, during the onset of the Brasiliano orogeny (650-620 Ma).
\end{abstract}

KEYWORDS: Pernambuco-Alagoas Domain; calc-alkaline orogenic granites; crystallization conditions; magmatic epidote; high ascension rates.

\section{INTRODUCTION}

Mineral and whole-rock chemistries constitute an important way to assess intensive crystallization parameters and to give information on the nature, source, and evolution of the magmas. These parameters (P-T- $\mathrm{fO}_{2}$-time path) are of first-order importance to comprehend the physicochemical evolution of granitic magmas. Studies on mineral chemistry of natural and experimental assemblages showed that ferromagnesian and coexisting mineral phases constitute a tool for estimating intensive crystallization parameters of granites (e.g., Anderson et al.2008). For example, biotite composition has been largely used as a redox and tectonomagmatic indicator (e.g., Wones and Eugster 1965, Nachit et al. 1985, Abdel-Rahman 1994, Shabani et al. 2003; among others). Calcic amphiboles are of special interest due to their compositional diversity and common occurrence, which constitute a good potential to investigate magmatic processes (Putirka 2016, and references therein). This phase has been successfully employed as a geobarometer and, together with plagioclase (the pair amphibole-plagioclase), as a powerful geothermometer (e.g., Hammarstron and Zen 1986, Hollister et al. 1987, Schmidt 1992, Holland

\footnotetext{
${ }^{1}$ Universidade Federal de Pernambuco - Recife (PE), Brazil. E-mails: rthyego@yahoo.com, valderez.ferreira@ufpe.br, mariucha_geo@yahoo.com.br, alcides.sial@ufpe.br ${ }^{*}$ Corresponding author.
}

and Blundy 1994, Mutch et al. 2016, among others). Titanite shows potential to quantitative estimates of pressure and temperature (e.g., Enami et al. 1993). Magmatic epidote (mEp) was initially described as indicating high-pressures of crystallization in intermediate calc-alkaline granites (>0.5 GPa; Zen and Hammarstrom 1984). However, the crystallization of this phase is strongly dependent on oxygen fugacity and bulk magma composition. Thus, at $\mathrm{fO}_{2}$ buffered by hematite-magnetite (HM) the stability of epidote is shifted to a lower pressure (>0.3 GPa; Schmidt and Thompson 1996, Ferreira et al. 2003, Schmidt and Poli 2004). Furthermore, this phase has been successfully employed to estimate the ascension rate of epidote-bearing magmas (e.g., Sial et al. 1999, 2008, Brasilino et al. 2011).

The Borborema Province (BP), in northeastern Brazil, comprises several intermediate plutonic rock associations that were recognized as containing epidote along with the main mafic coexisting mineral assemblage (biotite, amphibole, titanite) (e.g., Sial 1990, 1993, Brasilino et al. 1999, Sial et al. 1999, 2008, Ferreira et al. 2011, Long et al. 2005, 2019, Sial and Ferreira 2015, Campos et al. 2016). Therefore, it constitutes an important site for mineralogical studies using mineral chemistry, which allows estimating intensive crystallization parameters, including magma ascension rate. However, there is limited work regarding intensive crystallization parameters of plutonic rocks in the BP, especially in the Pernambuco-Alagoas Domain, where the presence of $\mathrm{mEp}$ was recently reported (cf. Silva et al., 2015, 2016, 2019, Ferreira et al. 2015, Silva 2017). 
In this work, we dealt with chemical data from biotite, hornblende, plagioclase, titanite, and epidote from the Major Isidoro and Monteirópolis epidote-bearing granites to investigate the physicochemical conditions $\left(\mathrm{P}-\mathrm{T}-\mathrm{fO}_{2}\right.$-time path-transport) in which they were generated.

\section{GEOLOGICAL BACKGROUND}

The Borborema Province, northeastern Brazil, is characterized by widespread Neoproterozoic-Cambrian granitic magmatism whose emplacement in many places is associated with shear zones (Santos and Medeiros 1999, Neves et al. 2006, Van Schmus et al. 2008, Ferreira et al. 1998, 2004, Amorim et al. 2019, Guimarães et al. 2004, Lima et al. 2017). The province is characterized by a mosaic of shear zones that are used to identify six main domains; one of them named Pernambuco-Alagoas (Fig. 1).Within this domain three granite series were identified: calc-alkaline, high-K calc-alkaline, and shoshonitic (e.g., Silva Filho et al. 2002, 2016, Brito et al. 2009, Ferreira et al. 2015). Silva Filho et al. (2014) divided the Pernambuco-Alagoas Domain into the Garanhuns, Água Branca, and Palmares subdomains. Migmatitic gneiss and granitic rocks of the Garanhuns sub-domain (northern portion) are derived predominantly from older (Paleoproterozoic) sources, whereas the others subdomains (southern portion) contain rocks derived from substantially younger (Tonian) sources. The Buíque-Paulo Afonso, Águas Belas-Canindé, and Ipojuca-Atalaia granitic batholiths (Silva Filho et al. 2002), each consisting of various plutons, which intruded Paleoproterozoic gneisses, represent these younger sources of the southern portion of the Água Branca and Palmares subdomains. This study focuses on part of the Águas Belas-Canindé (Água Branca subdomain).

\section{Field relationships and petrography}

The studied plutons constitute NE-SW elongate intrusions along the boundary of the Pernambuco-Alagoas and Sergipano domains, limited to the south/southeast by the Jacaré dos Homens transpressional shear zone (JHSZ) (Fig. 2) (De Oliveira 2008, Mendes et al. 2009, Lima et al. 2014). The Major Isidoro pluton is in sharp contact to the north with the Poço da Cacimba, and to the west with the Monteirópolis plutons (Silva et al. 2016, Lima 2019). The Major Isidoro pluton presents two main facies: biotite granite and epidote-amphibole-biotite tonalite, which consist of pink-gray leucocratic to mesocratic, coarse-grained to porphyritic with alkali-feldspar up to $5 \mathrm{~cm}$ in length. It shows a well-developed magmatic low angle dipping foliation that usually strikes northwest. However, in some samples, superposition of high-temperature

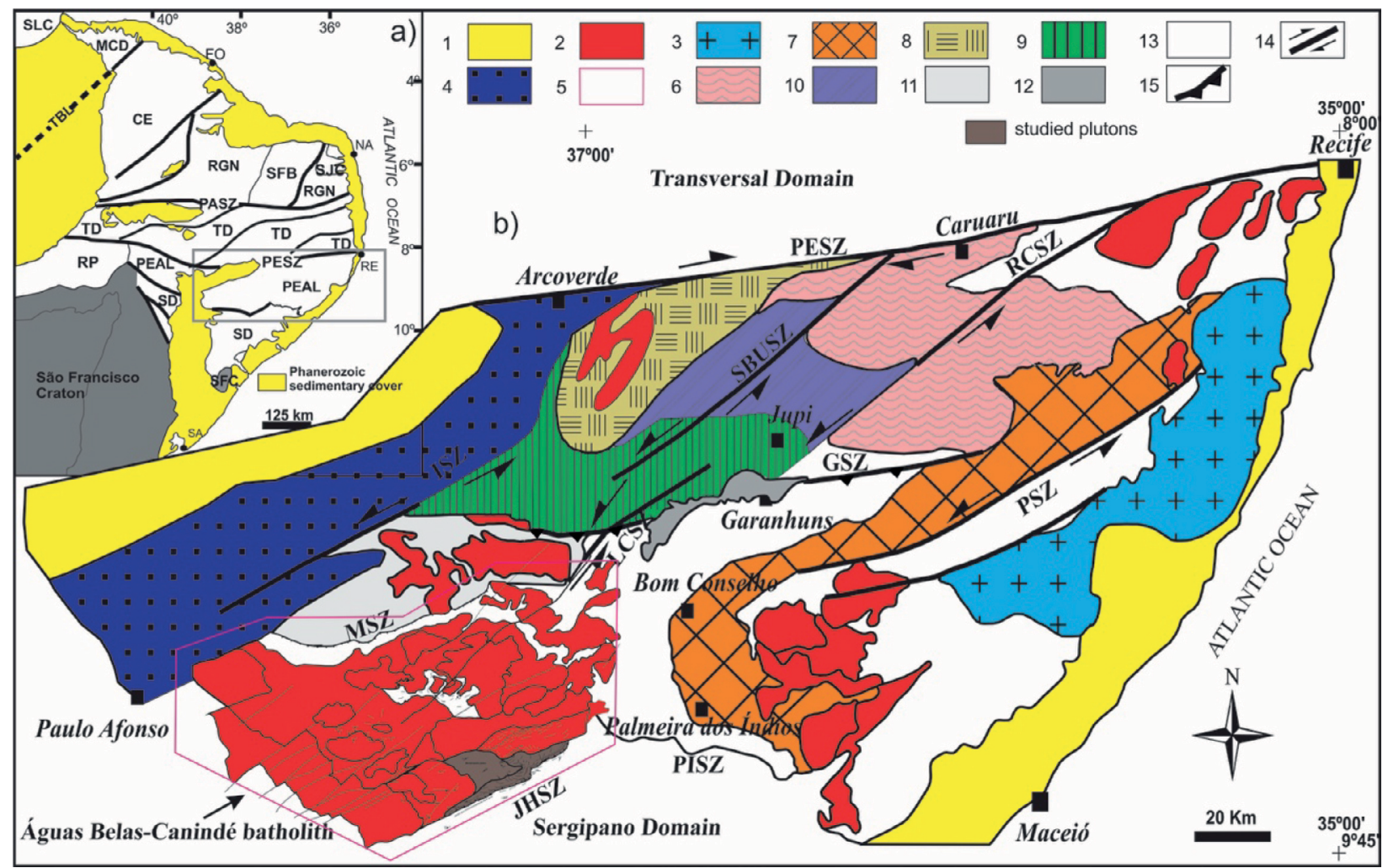

1: Phanerozoic sedimentary cover; 2: other Brasiliano granitoids; 3: Ipojuca-Atalaia batholith; 4: Buíque-Paulo Afonso batholith; 5: Águas Belas-Canindé batholith; 6: Granitoids and orthogneisses; 7: Palmares Sequence; 8: Rio Una (Unit 1) Sequence; 9: Rio Una (Unit 2) Sequence; 10: Rio Una (Unit 3) Sequence; 11: Inhapi Sequence; 12: Garanhuns quartzites; 13: basement-migmatites; 14: transcurrent shear zones (PESZ: Pernambuco; RCSZ: Rio da Chata; PSZ: Palmares; LCSZ: Limitão-Caetés; ISZ: Itaíba; SBUSZ: São Bento do Una); 15: compressive shear zones (GSZ: Garanhuns; MSZ: Maravilha; ZCJH: Jacaré dos Homens)

Figure 1. (A) Borborema Province. Major domains: MCD: Médio Coreaú; CE: Ceará; RGN: Rio Grande do Norte (SFB: Seridó Fold Belt; SJC: São José do Campestre Archaean nucleus); TD: Transverse; PEAL: Pernambuco-Alagoas; RP: Riacho do Pontal; SD: Sergipano; SFC: São Francisco Craton; SLC: São Luís Craton; Faults and shear zones: PASZ: Patos shear zone; PESZ: Pernambuco shear zone; TBL: Transbrasiliano Lineament. Cities and towns: Fo: Fortaleza; Na: Natal; Re: Recife; Sa: Salvador (Van Schmus et al. 2008). (B) Geologic map of the Pernambuco-Alagoas Domain (modified from Silva Filho et al. 2002, 2016). 
solid-state deformation expressed by chessboard extinction in quartz, development of myrmekitic intergrowths around alkali feldspar, and static recrystallization of feldspar grains occur. Elliptical diorite enclaves up to 0.5 meters long occur everywhere into this pluton; in some places, they appear along with the granite magmatic foliation, implying that they represent disrupted syn-plutonic dikes.

The Major Isidoro granite has undergone partial melting, segregation, and ductile deformation that suggest highstrain synkinematic emplacement. This pluton was emplaced coevally with an amphibolite facies metamorphic event in the region. In some parts, the granite is migmatized, where diatexite migmatite ( $c f$. Sawyer 2000, 2008), which shows up to 1 -meter heterogeneous schlieren features, are more common than metatexite migmatite; in this later, magmatic foliation is preserved. The schlierens in the diatexites are gray, characterized by biotite-rich domains separated by quartz-feldspar domains.

The Monteirópolis pluton consists of leucocratic mediumto coarse-grained amphibole-biotite alkali feldspar granodiorite to granite; porphyritic granite that displays up to $4.5 \mathrm{~cm}$ long zoned alkali feldspar crystals is also found. This pluton shows magmatic foliation that has dominantly gentle dips (usually $<30^{\circ}$, but sometimes up to $55^{\circ}$ ) to the northwest. Diorite enclaves and amphibole-rich clots, typically a few centimeters to about $25 \mathrm{~cm}$ long, are present mainly in the eastern portion of the pluton. These enclaves have sharp contacts with the host granite and show regular, generally oval, but occasionally lobate, with rare diffuse contours.

The mineral assemblage of quartz + alkali feldspar + plagioclase + biotite \pm hornblende + titanite \pm alanite \pm magnetite \pm ilmenite \pm epidote is common within both investigated plutons. Biotite and amphibole are the main mafic phases, and together with the shape-preferred orientation of alkali feldspar define the magmatic/metamorphic foliation of these plutons. Minor saussurite is observed as products of post-magmatic transformations of feldspars.

\section{AGE AND SR-ND-O ISOTOPIC SIGNATURES}

Silva et al. $(2015,2016)$ presented an integrated study of $\mathrm{Sr}$, $\mathrm{Nd}, \mathrm{Pb}$, and $\mathrm{O}$ isotopes for the studied intrusions. U-Pb SHRIMP zircon crystallization ages of the Major Isidoro and Monteirópolis batholiths are respectively $626.6 \pm 4.1$ and $625.8 \pm 3.7 \mathrm{Ma}$. Inherited zircon cores from the Major Isidoro yielded ages varying from 800 to $1,000 \mathrm{Ma}$. These crystallization ages point to an emplacement of the Major Isidoro and Monteirópolis batholiths to early stages of the Brasiliano orogeny. The Sr-Nd-O isotopic signatures constrain their sources. The Major Isidoro granites have $\mathrm{Nd}$-model ages of 1.1 to 1.4 , showing $\varepsilon \mathrm{Nd}_{(627 \mathrm{Ma})}$ ${ }^{87} \mathrm{Sr} /{ }^{86} \mathrm{Sr}_{(627 \mathrm{Ma})}$ and $\delta^{18} \mathrm{O}_{\text {(zircon) }}$ (-1.09 to $-2.12,0.7069$ to 0.7086 , and 6.95 to $7.02 \%$, respectively) more evolved than the Monteirópolis granites that show homogeneous model age of $1.0 \mathrm{Ga}$ and higher $\varepsilon \mathrm{Nd}_{(625 \mathrm{Ma})}$, and lower ${ }^{87} \mathrm{Sr} /{ }^{86} \mathrm{Sr}_{(625 \mathrm{Ma})}$ and $\delta^{18} \mathrm{O}_{\text {(zircon) }}(-0.78$ to $+1.06,0.7050-0.7052,5.0$ to $5.9 \%$ o).

\section{ANALYTICAL PROCEDURES}

All samples for whole-rock chemical analyses were crushed in a stainless steel jaw crusher and split to $<74 \mu \mathrm{m}$ grain size in a chromium steel ring mill. The Loss On Ignition (LOI) was determined on one gram of a pre-dried sample by igniting to $1,000^{\circ} \mathrm{C}$ for two hours. Major and some trace elements $(\mathrm{Sr}, \mathrm{Zr}$, $\mathrm{Nb}, \mathrm{Y}, \mathrm{Rb}, \mathrm{Ba}$, and $\mathrm{Ni}$ ) were determined on fused discs of sample

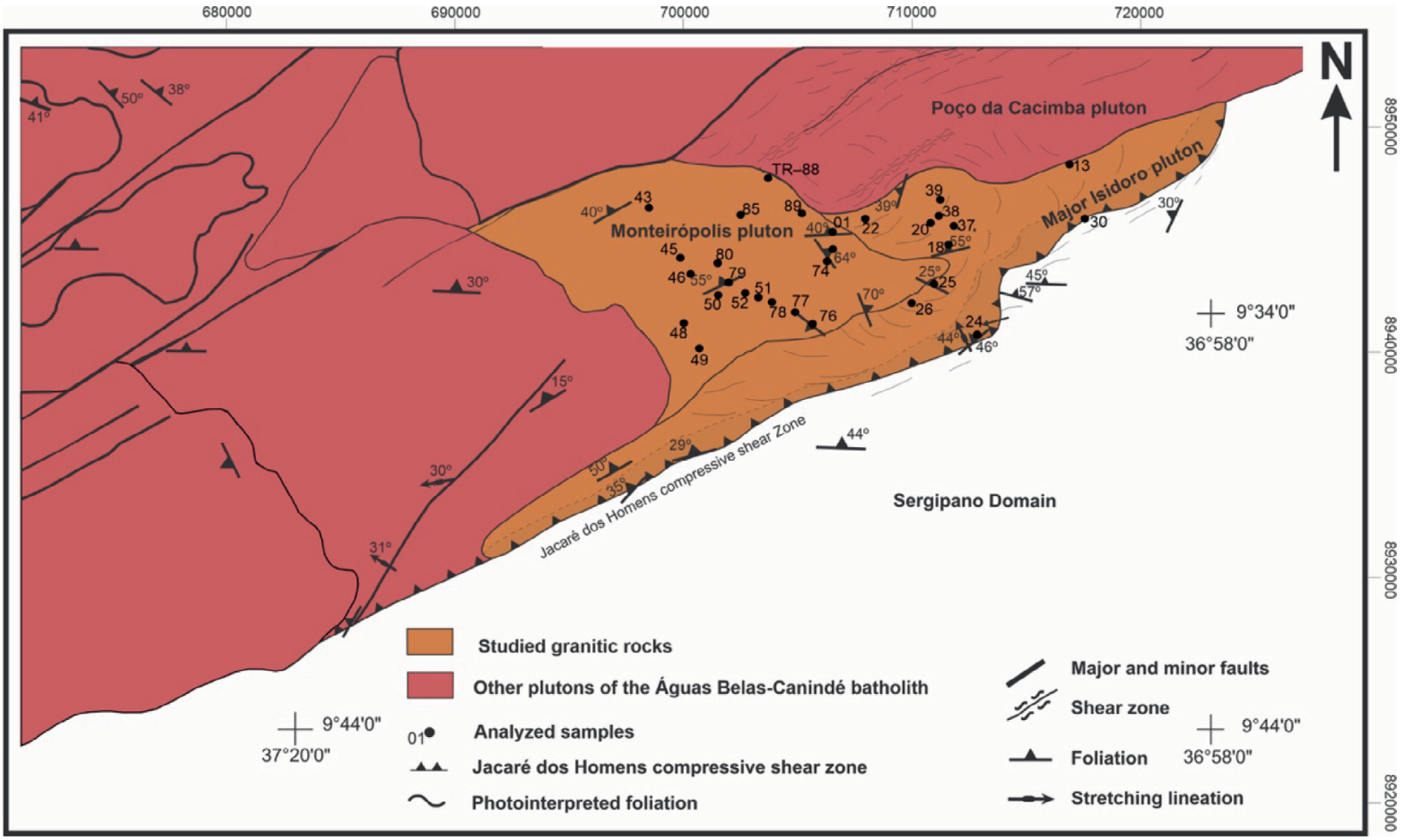

Figure 2. Geological map of the Águas Belas-Canindé batholith showing the studied plutons. 
mixed with lithium tetraborate flux (ratio sample: flux $=1: 5$ fused in a Pt-Au crucible) with a fully automated Rigaku RIX3000 XRF spectrometer at the NEG-LABISE, Universidade Federal de Pernambuco, Recife, Brazil. The calibration curves were prepared by analyses of international reference materials (AC-E, AL-I, AN-G, BE-N, IF-G, and MA-N from the International Working Group (IWG), DR-N, FK-N, and UB-N from the Association Nationale de la Recherche et de la Technologie (ANRT), GA, GH, MICA-MG, PM-S, and WS-E from the Centre de Recherches Pétrographiques et Géochimiques (CRPG).

Mineral chemistry analyses were performed at the Electron Microprobe Laboratory, Universidade de Brasilia, Brazil, using a Superprobe JEOL JXA-8230 equipped with WDS, with an accelerating potential of $15 \mathrm{kV}$, a current of $10 \eta \mathrm{A}$, and a $5 \mu$ diameter beam. WDS spot analyses of the main rock-forming minerals were performed using the following standards and X-ray lines: albite $(\mathrm{Na} K \alpha)$, forsterite $(\mathrm{Mg} \mathrm{K} \alpha)$, topaz $(\mathrm{F} \mathrm{K} \alpha)$, microcline $(\mathrm{Al} \mathrm{K} \alpha, \mathrm{Si} K \alpha, \mathrm{K} K \alpha)$, andradite $(\mathrm{Ca} \mathrm{K} \alpha)$, vanadinite (V K $\alpha, \mathrm{Cl} \mathrm{K} \alpha), \mathrm{MnTiO}_{3}(\mathrm{Ti} \mathrm{K} \alpha, \mathrm{Mn} \mathrm{K} \alpha), \mathrm{Cr}_{2} \mathrm{O}_{3}(\mathrm{Cr} \mathrm{K} \alpha)$,
$\mathrm{SrSO}_{4}(\mathrm{Sr} \mathrm{L} \alpha), \mathrm{NiO}(\mathrm{Ni} \mathrm{K} \alpha)$, andradite $(\mathrm{Fe} \mathrm{K} \alpha)$, and $\mathrm{BaSO}_{4}$ ( $\mathrm{Ba} L \alpha)$. Counting times were 10 s on peak and 5 s on the two background positions for all elements. Matrix effects corrections were computed following the PRZ procedure and raw data reduction was done with Armstrong software from JEOL. Cationic proportions and structural formulae of plagioclase, amphibole, titanite, biotite, and epidote were calculated based on 32, 23, 5, 22 and 12.5 oxygens in the formula unit, respectively (Deer et al. 2013). Tables 1 to 5 contain representative microprobe chemical analyses.

\section{MINERAL CHEMISTRY}

\section{Plagioclase}

Microprobe analyses of plagioclase from the Major Isidoro granites and enclaves indicate compositions between $\mathrm{An}_{23-35}$ (oligoclase-andesine) and $\mathrm{An}_{34-40}$ (andesine), respectively (Fig. 3; Tab. 1). Plagioclase from the Monteirópolis granite

Table 1. Microprobe analyses for plagioclase of the studied granites.

\begin{tabular}{|c|c|c|c|c|c|c|c|c|c|c|}
\hline Sample & TR-89 & TR-89 & TR-89 & TR-89 & TR-22 & TR-22 & TR-22 & TR-22 & TR-30 & TR-30 \\
\hline Location & Core & Rim & Core & Rim & Core & Rim & Core & Rim & Core & Rim \\
\hline $\mathrm{SiO}_{2}$ & 64.44 & 64.41 & 64.40 & 64.65 & 61.41 & 62.28 & 58.95 & 61.06 & 57.58 & 59.50 \\
\hline $\mathrm{TiO}_{2}$ & 0.03 & 0.03 & bdl & bdl & 0.06 & 0.02 & 0.02 & bdl & bdl & bdl \\
\hline $\mathrm{CaO}$ & 3.53 & 3.45 & 4.03 & 3.31 & 5.18 & 4.78 & 7.31 & 6.02 & 8.20 & 7.05 \\
\hline $\mathrm{Al}_{2} \mathrm{O}_{3}$ & 22.19 & 22.17 & 22.63 & 21.88 & 23.67 & 22.98 & 25.00 & 23.94 & 26.07 & 25.08 \\
\hline $\mathrm{Na}_{2} \mathrm{O}$ & 9.35 & 9.37 & 9.08 & 9.55 & 8.64 & 8.78 & 7.30 & 8.22 & 6.96 & 7.73 \\
\hline $\mathrm{FeO}$ & 0.04 & 0.07 & 0.01 & bdl & 0.07 & 0.12 & 0.03 & 0.33 & 0.15 & 0.20 \\
\hline $\mathrm{MgO}$ & 0.00 & 0.02 & bdl & bdl & bdl & bdl & bdl & 0.01 & 0.01 & bdl \\
\hline $\mathrm{K}_{2} \mathrm{O}$ & 0.18 & 0.14 & 0.13 & 0.19 & 0.26 & 0.24 & 0.11 & 0.15 & 0.16 & 0.17 \\
\hline $\mathrm{MnO}$ & bdl & bdl & 0.03 & bdl & bdl & 0.03 & bdl & bdl & bdl & 0.01 \\
\hline $\mathrm{SrO}$ & 0.11 & 0.32 & 0.02 & 0.22 & bdl & 0.16 & 0.18 & bdl & 0.09 & 0.12 \\
\hline $\mathrm{BaO}$ & bdl & 0.03 & 0.10 & 0.02 & 0.10 & bdl & bdl & bdl & 0.07 & bdl \\
\hline Total & 99.88 & 100.01 & 100.43 & 99.82 & 99.39 & 99.40 & 98.89 & 99.72 & 99.31 & 99.85 \\
\hline \multicolumn{11}{|c|}{ Cation proportions on the basis of 32 oxygens } \\
\hline $\mathrm{Si}$ & 2.843 & 2.843 & 2.828 & 2.856 & 2.745 & 2.780 & 2.661 & 2.725 & 2.600 & 2.662 \\
\hline $\mathrm{Ti}$ & 0.001 & 0.001 & - & - & 0.002 & 0.001 & 0.001 & - & - & - \\
\hline $\mathrm{Ca}$ & 0.167 & 0.163 & 0.190 & 0.157 & 0.248 & 0.228 & 0.353 & 0.288 & 0.397 & 0.338 \\
\hline $\mathrm{Al}^{\text {tot }}$ & 1.154 & 1.154 & 1.171 & 1.139 & 1.247 & 1.209 & 1.330 & 1.259 & 1.387 & 1.322 \\
\hline $\mathrm{Na}$ & 0.800 & 0.802 & 0.773 & 0.818 & 0.749 & 0.760 & 0.639 & 0.712 & 0.609 & 0.670 \\
\hline $\mathrm{Fe}$ & 0.002 & 0.002 & - & - & 0.003 & 0.004 & 0.001 & 0.012 & 0.006 & 0.007 \\
\hline $\mathrm{Mg}$ & - & - & - & - & - & - & - & 0.001 & 0.001 & - \\
\hline K & 0.010 & 0.008 & 0.007 & 0.011 & 0.015 & 0.013 & 0.007 & 0.009 & 0.009 & 0.009 \\
\hline $\mathrm{Mn}$ & - & - & 0.001 & - & - & 0.001 & - & - & - & - \\
\hline $\mathrm{Sr}$ & 0.003 & 0.008 & 0.001 & 0.006 & - & 0.004 & 0.005 & - & 0.002 & 0.003 \\
\hline $\mathrm{Ba}$ & - & 0.001 & 0.002 & - & 0.002 & - & - & - & 0.001 & - \\
\hline Sum & 4.983 & 4.984 & 4.976 & 4.988 & 5.011 & 5.002 & 4.996 & 5.005 & 5.017 & 5.016 \\
\hline mol.\% An & 16.69 & 16.31 & 18.96 & 15.66 & 24.79 & 22.84 & 35.34 & 28.77 & 39.69 & 33.77 \\
\hline $\mathrm{mol} . \% \mathrm{Ab}$ & 79.98 & 80.21 & 77.26 & 81.83 & 74.88 & 76.00 & 63.87 & 71.16 & 60.91 & 67.01 \\
\hline mol. \% Or & 1.04 & 0.78 & 0.73 & 1.05 & 1.49 & 1.34 & 0.65 & 0.86 & 0.94 & 0.94 \\
\hline
\end{tabular}

bdl: below detection limit; TR-22: Major Isidoro granite; TR-30: Major Isidoro enclave; TR-89: Monteirópolis granite. 
Braz. J. Geol. (2020), 50(2): e20190110

Table 2. Microprobe analyses and structural formulae for amphibole of the studied granites*.

\begin{tabular}{|c|c|c|c|c|c|c|c|c|c|c|c|c|c|}
\hline Sample & $\begin{array}{c}\text { TR- } \\
89 \text { C2 }\end{array}$ & $\begin{array}{c}\text { TR- } \\
89 \text { C2 }\end{array}$ & $\begin{array}{c}\text { TR- } \\
89 \text { C2 }\end{array}$ & $\begin{array}{c}\text { TR- } \\
89 \_ \text {C2 }\end{array}$ & $\begin{array}{c}\text { TR- } \\
89 \text { C5 }\end{array}$ & $\begin{array}{c}\text { TR- } \\
89 \text { C5 }\end{array}$ & $\begin{array}{c}\text { TR- } \\
\text { 22_C5 }\end{array}$ & $\begin{array}{c}\text { TR- } \\
\text { 22_C5 }\end{array}$ & $\begin{array}{c}\text { TR- } \\
22 \_ \text {C3 }\end{array}$ & $\begin{array}{c}\text { TR- } \\
\text { 22_C3 }\end{array}$ & $\begin{array}{c}\text { TR- } \\
22 \_C 2\end{array}$ & $\begin{array}{c}\text { TR- } \\
\text { 30_C1 }\end{array}$ & $\begin{array}{c}\text { TR- } \\
\text { 30_C1 }\end{array}$ \\
\hline Location & Core & Rim & Core & Rim & Core & Rim & Core & Rim & Core & Rim & Rim & Core & Rim \\
\hline $\mathrm{SiO}_{2}$ & 43.573 & 43.361 & 43.269 & 42.743 & 43.942 & 44.12 & 42.114 & 41.355 & 41.047 & 42.145 & 41.519 & 41.25 & 41.603 \\
\hline $\mathrm{TiO}_{2}$ & 0.926 & 0.93 & 1.025 & 0.49 & 0.592 & 0.632 & 1.067 & 1.065 & 0.672 & 0.877 & 0.377 & 1.227 & 1.445 \\
\hline $\mathrm{Al}_{2} \mathrm{O}_{3}$ & 8.913 & 8.83 & 8.833 & 9.545 & 9.02 & 9.19 & 10.211 & 10.471 & 11.104 & 10.806 & 12.898 & 11.776 & 11.438 \\
\hline $\mathrm{FeO}^{*}$ & 19.885 & 20.21 & 20.219 & 20.478 & 20.043 & 19.926 & 20.528 & 20.528 & 21.7 & 20.975 & 22.08 & 19.192 & 18.791 \\
\hline $\mathrm{MgO}$ & 8.984 & 8.99 & 9.101 & 8.493 & 9.465 & 9.09 & 8.447 & 8.379 & 7.75 & 7.715 & 7.013 & 9.153 & 8.959 \\
\hline $\mathrm{MnO}$ & 0.742 & 0.643 & 0.714 & 0.614 & 0.607 & 0.844 & 0.594 & 0.592 & 0.697 & 0.735 & 0.772 & 0.309 & 0.489 \\
\hline $\mathrm{CaO}$ & 11.349 & 11.102 & 11.219 & 11.399 & 11.337 & 11.176 & 11.264 & 11.311 & 11.488 & 11.482 & 11.362 & 11.933 & 11.733 \\
\hline $\mathrm{Na}_{2} \mathrm{O}$ & 1.691 & 1.637 & 1.697 & 1.6 & 1.548 & 1.655 & 1.394 & 1.426 & 1.414 & 1.429 & 1.356 & 1.216 & 1.406 \\
\hline $\mathrm{K}_{2} \mathrm{O}$ & 1.249 & 1.281 & 1.245 & 1.297 & 1.178 & 1.32 & 1.36 & 1.36 & 1.362 & 1.394 & 1.395 & 1.666 & 1.666 \\
\hline $\mathrm{F}$ & 0.48 & 0.43 & 0.426 & 0.458 & 0.532 & 0.539 & 0.298 & 0.204 & 0.285 & 0.216 & 0.318 & 0.231 & 0.185 \\
\hline $\mathrm{Cl}$ & 0.024 & 0.017 & 0.045 & 0.021 & 0.007 & 0.018 & 0.04 & 0.059 & 0.062 & 0.027 & 0.073 & 0.053 & 0.053 \\
\hline $\mathrm{H}_{2} \mathrm{O}^{* *}$ & 1.72 & 1.73 & 1.73 & 1.71 & 1.71 & 1.71 & 1.78 & 1.81 & 1.77 & 1.83 & 1.79 & 1.83 & 1.86 \\
\hline $\mathrm{OH} \equiv(\mathrm{F}, \mathrm{Cl})$ & 0.21 & 0.18 & 0.19 & 0.20 & 0.23 & 0.23 & 0.13 & 0.10 & 0.13 & 0.10 & 0.15 & 0.11 & 0.09 \\
\hline Total & 99.32 & 98.98 & 99.34 & 98.65 & 99.75 & 99.98 & 98.97 & 98.46 & 99.22 & 99.54 & 100.80 & 99.73 & 99.53 \\
\hline \multicolumn{14}{|c|}{ Structural formulae on the basis of 23 oxygens } \\
\hline \multicolumn{14}{|l|}{ T-sites } \\
\hline $\mathrm{Si}$ & 6.639 & 6.624 & 6.590 & 6.565 & 6.624 & 6.660 & 6.434 & 6.356 & 6.283 & 6.432 & 6.246 & 6.225 & 6.309 \\
\hline$A l^{\text {iv }}$ & 1.361 & 1.376 & 1.410 & 1.435 & 1.376 & 1.340 & 1.566 & 1.644 & 1.717 & 1.568 & 1.754 & 1.775 & 1.691 \\
\hline $\mathrm{Al}($ total) & 1.601 & 1.590 & 1.586 & 1.728 & 1.603 & 1.636 & 1.839 & 1.897 & 2.004 & 1.944 & 2.288 & 2.095 & 2.045 \\
\hline \multicolumn{14}{|l|}{ M1,2,3 sites } \\
\hline $\mathrm{Al}^{\mathrm{vi}}$ & 0.240 & 0.214 & 0.175 & 0.293 & 0.227 & 0.296 & 0.274 & 0.253 & 0.287 & 0.376 & 0.534 & 0.320 & 0.354 \\
\hline $\mathrm{Ti}$ & 0.106 & 0.107 & 0.117 & 0.057 & 0.067 & 0.072 & 0.123 & 0.123 & 0.077 & 0.101 & 0.043 & 0.139 & 0.165 \\
\hline $\mathrm{Fe}^{3+}$ & 0.431 & 0.516 & 0.544 & 0.537 & 0.621 & 0.475 & 0.642 & 0.707 & 0.820 & 0.534 & 0.757 & 0.682 & 0.478 \\
\hline $\mathrm{Mg}$ & 2.040 & 2.047 & 2.066 & 1.944 & 2.126 & 2.045 & 1.923 & 1.919 & 1.768 & 1.755 & 1.572 & 2.059 & 2.025 \\
\hline $\mathrm{Mn}$ & 0.096 & 0.083 & 0.092 & 0.080 & 0.078 & 0.108 & 0.077 & 0.077 & 0.090 & 0.095 & 0.098 & 0.039 & 0.063 \\
\hline $\mathrm{Fe}^{2+}$ & 2.088 & 2.034 & 2.005 & 2.089 & 1.880 & 2.005 & 1.962 & 1.921 & 1.957 & 2.140 & 1.995 & 1.740 & 1.906 \\
\hline $\mathrm{Ca}$ & 0.000 & 0.000 & 0.000 & 0.000 & 0.000 & 0.000 & 0.000 & 0.000 & 0.000 & 0.000 & 0.000 & 0.021 & 0.010 \\
\hline Sum & 5.000 & 5.000 & 5.000 & 5.000 & 5.000 & 5.000 & 5.000 & 5.000 & 5.000 & 5.000 & 5.000 & 5.000 & 5.000 \\
\hline \multicolumn{14}{|l|}{ M4 site } \\
\hline $\mathrm{Fe}$ & 0.015 & 0.032 & 0.026 & 0.004 & 0.026 & 0.036 & 0.020 & 0.011 & 0.001 & 0.004 & 0.025 & 0.000 & 0.000 \\
\hline $\mathrm{Ca}$ & 1.853 & 1.817 & 1.831 & 1.876 & 1.831 & 1.808 & 1.844 & 1.863 & 1.884 & 1.878 & 1.832 & 1.909 & 1.897 \\
\hline $\mathrm{Na}$ & 0.132 & 0.151 & 0.143 & 0.120 & 0.143 & 0.156 & 0.136 & 0.127 & 0.115 & 0.119 & 0.143 & 0.091 & 0.103 \\
\hline Sum & 2.000 & 2.000 & 2.000 & 2.000 & 2.000 & 2.000 & 2.000 & 2.000 & 2.000 & 2.000 & 2.000 & 2.000 & 2.000 \\
\hline \multicolumn{14}{|l|}{ A site } \\
\hline $\mathrm{Ca}$ & 0.000 & 0.000 & 0.000 & 0.000 & 0.000 & 0.000 & 0.000 & 0.000 & 0.000 & 0.000 & 0.000 & 0.000 & 0.000 \\
\hline $\mathrm{Na}$ & 0.368 & 0.334 & 0.358 & 0.357 & 0.309 & 0.328 & 0.277 & 0.298 & 0.304 & 0.304 & 0.253 & 0.265 & 0.310 \\
\hline $\mathrm{K}$ & 0.243 & 0.250 & 0.242 & 0.254 & 0.227 & 0.254 & 0.265 & 0.267 & 0.266 & 0.271 & 0.268 & 0.321 & 0.322 \\
\hline Sum A & 0.611 & 0.584 & 0.600 & 0.611 & 0.536 & 0.582 & 0.542 & 0.565 & 0.570 & 0.576 & 0.520 & 0.585 & 0.632 \\
\hline $\mathrm{OH}$ & 1.760 & 1.785 & 1.781 & 1.769 & 1.741 & 1.735 & 1.843 & 1.884 & 1.843 & 1.887 & 1.827 & 1.874 & 1.897 \\
\hline $\mathrm{F}$ & 0.233 & 0.210 & 0.208 & 0.225 & 0.257 & 0.260 & 0.146 & 0.101 & 0.140 & 0.105 & 0.154 & 0.112 & 0.090 \\
\hline $\mathrm{Cl}$ & 0.006 & 0.004 & 0.012 & 0.006 & 0.002 & 0.005 & 0.011 & 0.016 & 0.016 & 0.007 & 0.019 & 0.014 & 0.014 \\
\hline Sum & 2.000 & 2.000 & 2.000 & 2.000 & 2.000 & 2.000 & 2.000 & 2.000 & 2.000 & 2.000 & 2.000 & 2.000 & 2.000 \\
\hline $\mathrm{Mg} /\left(\mathrm{Mg}+\mathrm{Fe}^{2+}\right)$ & 0.492 & 0.498 & 0.504 & 0.481 & 0.527 & 0.501 & 0.493 & 0.498 & 0.474 & 0.450 & 0.438 & 0.542 & 0.515 \\
\hline $\begin{array}{l}\mathrm{T} \text { (Blundy and } \\
\text { Holland 1990) }\end{array}$ & 720 & 725 & 734 & 728 & 719 & 707 & 770 & 791 & 806 & 761 & 787 & 879 & 842 \\
\hline $\begin{array}{l}\text { T (Holland and } \\
\text { Blundy 1994) }\end{array}$ & 724 & 732 & 758 & 714 & 722 & 692 & 740 & 766 & 767 & 712 & 695 & 778 & 765 \\
\hline $\begin{array}{l}\mathrm{T} \text { (Holland and } \\
\text { Blundy 1994) }\end{array}$ & 672 & 685 & 696 & 662 & 672 & 660 & 710 & 720 & 716 & 684 & 686 & 735 & 730 \\
\hline $\begin{array}{l}P \text { (Anderson and } \\
\text { Smith 1995) }\end{array}$ & 4.64 & 4.46 & 4.30 & 5.34 & 4.65 & 4.92 & 5.27 & 5.36 & 5.91 & 6.14 & 7.71 & 5.96 & 5.83 \\
\hline $\mathrm{P}$ (Schmidt 1992) & 4.61 & 4.56 & 4.54 & 5.22 & 4.62 & 4.78 & 5.74 & 6.02 & 6.53 & 6.24 & 7.88 & 6.96 & 6.72 \\
\hline
\end{tabular}

*Temperature in ${ }^{\circ} \mathrm{C}$ calculated after Blundy and Holland (1990) and Holland and Blundy (1994) reaction A; reaction B); pressure in kbar calculated after Anderson and Smith (1995) and Schmidt (1992);. TR-22: Major Isidoro granite; TR-30: Major Isidoro enclave; TR-89: Monteirópolis granite; ${ }^{*}$ total iron; ${ }^{* *}$ calculated. 
has relatively homogeneous compositions varying from $\mathrm{An}_{16}$ to $\mathrm{An}_{19}$ (oligoclase). Plagioclase grains from the two plutons shows normal zoning with the nuclei slightly enriched in $\mathrm{Ca}$ compared to rim presenting low $\mathrm{Ba}(0.002 \mathrm{apfu}$ [atoms per formula units]) and $\mathrm{Fe}^{3+}(0.012)$ contents.

\section{Amphibole}

Analyzed hornblende grains (Tab. 2) classified according to nomenclature scheme proposed by Leake et al. $(1997,2003)$ are members of the calcic group in which $(\mathrm{Ca}+\mathrm{Na})_{\mathrm{B}} \geq 1.50$ and $\mathrm{Na}_{\mathrm{B}} \leq 0.50$. Amphibole grains from the Major Isidoro granite and enclaves show $\mathrm{Si}$ content from 6.25 to 6.43 atoms per formula units (apfu), $\mathrm{Al}^{\mathrm{VI}}$ contents in the range of 0.25 to $0.534 \mathrm{apfu}$, and $\mathrm{Ti}$ content in the 0.04 to $0.12 \mathrm{apfu}$ range. Amphiboles from granites and enclaves are, respectively, hastingsite and Mg-hastingsite (Fig. 4). Amphibole grains from the Monteirópolis pluton have lower Si contents than those in the Major Isidoro granite, in the range of 6.56 to $6.66 \mathrm{apfu}, \mathrm{Al}^{\mathrm{VI}}$ contents in the range of 0.17 to $0.29 \mathrm{apfu}$, and Ti contents in a narrow range from 0.06 to $0.11 \mathrm{apfu}$; they are classified as Fe-edenite to edenite (Fig. 4). Based on the new classification and nomenclature scheme for the amphiboles (Hawthorne et al. 2012), all analyzed hornblendes classify as pargasite. Amphibole compositions were plotted (Figs. 5A and 5B) in order to test the role of Edenite and Tschermak exchanges. The relatively homogeneous compositions suggest that Edenite $\left(\mathrm{Al}^{\mathrm{VI}}<0.2 \mathrm{apfu}\right)$ and Tschermak $\left(\mathrm{Al}^{\mathrm{Tot}}<0.2 \mathrm{apfu}\right)$ exchange mechanisms play a minor role in the evolution of the two plutons.

\section{Titanite}

Titanite grains from the studied plutons show very low $\mathrm{Al}+\mathrm{Fe}^{3+}(<0.12$; Tabs. 3A and 3B). The composition of titanite depends on temperature and pressure (Enami et al. 1993). These authors suggested that titanite crystals having $\mathrm{Al}+\mathrm{Fe}^{3+}$ $<0.35$ apfu indicate crystallization at temperatures greater than $\sim 700^{\circ} \mathrm{C}$. Our results point to crystallization within the range for typical high-temperature titanite $\left(>700^{\circ} \mathrm{C}\right)$, which is consistent with the early crystallization of euhedral titanite in these plutons.

\section{Biotite}

Biotite from the Major Isidoro granites has higher $\mathrm{Al}^{\text {tot }}$ contents (2.67 to $2.94 \mathrm{apfu}$ and $\mathrm{Fe} \#(\mathrm{Fe} /(\mathrm{Mg}+\mathrm{Fe})$ of 0.50 to 0.58 ) compared to biotite in Monteirópolis granites, with $\mathrm{Al}^{\text {tot }}$ varying from 2.49 to $2.85 \mathrm{apfu}$, and Fe\# from 0.44 to 0.55 (Tabs. 4A and 4B). These compositions lying approximately between the annite and phlogopite end-members (Fig. 6). Such compositions are in the typical range from orogenic calc-alkaline granites when compared with those presented by Nachit et al. (1985) and Abdel-Rahman (1994) (Figs. 7, $8 \mathrm{~A}$ and $8 \mathrm{~B}$ ). Biotite crystals from both plutons have fluorine contents dominant over chlorine.

\section{Epidote}

According to chemical criteria of Tulloch (1979), Johnston and Wyllie (1988), and Vyhnal et al. (1991), magmatic epidote in granites has pistacite contents ( $\mathrm{Ps}=$ molar $\left.\left[\mathrm{Fe}^{3+} /\left(\mathrm{Fe}^{3+}+\mathrm{Al}\right)\right] \times 100\right)$ between 25 to $29 \mathrm{~mol} \%$; however, epidote grains described in the Borborema Province have Ps contents of up to $33 \mathrm{~mol} \%$ (Sial et al. 1999, 2008, Ferreira et al. 2003, 2011, 2015; among others), which enlarge typical Ps ranges to $25-33 \mathrm{~mol} \%$. The analyzed crystals of the Major Isidoro granite yield values of 27 to $29 \mathrm{~mol} \%$, while the analyzed grains of the Monteirópolis plutons show Ps values of 29 to $31 \mathrm{~mol} \%$, which are within the range (25-33 mol\%) proposed by these authors. Magmatic epidote, according to Evans and Vance (1987), has $\mathrm{TiO}_{2}$ contents < $0.6 \mathrm{wt} \%$, while secondary epidote replacing biotite has $\mathrm{TiO}_{2}$ contents $>0.6 \mathrm{wt} . \%$. The analyzed crystals present values ranging from 0.0 to $0.3 \mathrm{wt}$.\% for both plutons (Table 5), suggesting that they are primary, in accordance with the Tulloch's criteria based on pistacite contents in addition to textural evidence.

Table 3A. Microprobe analyses for titanite of the Major Isidoro granites.

\begin{tabular}{|c|c|c|c|c|c|c|}
\hline Sample & TR-13 & TR-13 & TR-22 & TR-22 & TR-22 & TR-22 \\
\hline Location & Core & Rim & Core & Rim & Core & Rim \\
\hline $\mathrm{SiO}_{2}$ & & 30.58 & 30.50 & 29.63 & 30.54 & 30.12 \\
\hline $\mathrm{TiO}_{2}$ & 35.32 & 36.51 & 35.01 & 35.65 & 35.90 & 37.13 \\
\hline $\mathrm{CaO}$ & 28.79 & 28.67 & 28.64 & 27.77 & 28.18 & 27.63 \\
\hline $\mathrm{Al}_{2} \mathrm{O}_{3}$ & 2.23 & 2.36 & 1.43 & 1.44 & 1.42 & 1.37 \\
\hline $\mathrm{Fe}_{2} \mathrm{O}_{3}$ & 0.73 & 0.73 & 1.21 & 1.43 & 1.27 & 1.42 \\
\hline $\mathrm{Na}_{2} \mathrm{O}$ & 0.04 & 0.03 & 0.05 & 0.04 & 0.03 & 0.02 \\
\hline $\mathrm{MgO}$ & 0.00 & 0.01 & 0.03 & 0.01 & 0.01 & 0.01 \\
\hline $\mathrm{V}_{2} \mathrm{O}_{3}$ & 0.33 & 0.37 & 0.38 & 0.36 & 0.29 & 0.33 \\
\hline $\mathrm{MnO}$ & 0.09 & 0.05 & 0.12 & 0.21 & 0.12 & 0.22 \\
\hline $\mathrm{BaO}$ & 0.01 & 0.07 & 0.21 & 0.19 & 0.12 & 0.08 \\
\hline F & 0.49 & 0.58 & 0.41 & 0.40 & 0.46 & 0.34 \\
\hline $\mathrm{H}_{2} \mathrm{O}^{* *}$ & 0.25 & 0.22 & 0.19 & 0.23 & 0.18 & 0.24 \\
\hline $\mathrm{OH} \equiv \mathrm{F}$ & 0.21 & 0.24 & 0.17 & 0.17 & 0.19 & 0.14 \\
\hline Total & 98.77 & 99.93 & 98.01 & 97.17 & 98.32 & 98.77 \\
\hline \multicolumn{7}{|c|}{ Cation proportions on the basis of 5 oxygens } \\
\hline $\mathrm{Si}$ & 1.019 & 1.004 & 1.022 & 1.003 & 1.020 & 1.001 \\
\hline $\mathrm{Ti}$ & 0.881 & 0.901 & 0.882 & 0.908 & 0.901 & 0.928 \\
\hline $\mathrm{Ca}$ & 1.023 & 1.008 & 1.028 & 1.008 & 1.008 & 0.984 \\
\hline $\mathrm{Al}$ & 0.087 & 0.091 & 0.056 & 0.058 & 0.056 & 0.054 \\
\hline $\mathrm{Fe}^{3+}$ & 0.018 & 0.018 & 0.030 & 0.036 & 0.032 & 0.035 \\
\hline $\mathrm{Na}$ & 0.002 & 0.002 & 0.003 & 0.002 & 0.002 & 0.001 \\
\hline $\mathrm{Mg}$ & 0.000 & 0.000 & 0.002 & 0.000 & 0.001 & 0.001 \\
\hline V & 0.009 & 0.010 & 0.010 & 0.010 & 0.008 & 0.009 \\
\hline $\mathrm{Mn}$ & 0.002 & 0.001 & 0.003 & 0.006 & 0.003 & 0.006 \\
\hline $\mathrm{Ba}$ & 0.000 & 0.001 & 0.003 & 0.002 & 0.002 & 0.001 \\
\hline Sum & 3.043 & 3.036 & 3.041 & 3.034 & 3.032 & 3.019 \\
\hline $\mathrm{F}$ & 0.051 & 0.060 & 0.043 & 0.043 & 0.049 & 0.036 \\
\hline $\mathrm{Al}+\mathrm{Fe}^{3+}$ & 0.106 & 0.109 & 0.087 & 0.094 & 0.088 & 0.089 \\
\hline $\begin{array}{l}\mathrm{Ti}^{4+} / \\
\left(\mathrm{Al}+\mathrm{Fe}^{3+}\right)\end{array}$ & 8.343 & 8.238 & 10.179 & 9.664 & 10.249 & 10.395 \\
\hline $\mathrm{OH}^{* *}$ & 0.054 & 0.049 & 0.043 & 0.051 & 0.039 & 0.053 \\
\hline $\mathrm{X}_{\mathrm{F}} \mathrm{g}$ & 0.48 & 0.55 & 0.50 & 0.46 & 0.55 & 0.40 \\
\hline
\end{tabular}

$\mathrm{XF}: \mathrm{F} /(\mathrm{F}+\mathrm{OH})$; $\mathrm{OH}$ calculated as $\mathrm{OH}=(\mathrm{Al}+\mathrm{Fe} 3+)-\mathrm{F}$. 
Braz. J. Geol. (2020), 50(2): e20190110

Table 3B. Microprobe analyses for titanite of the Monteirópolis granites.

\begin{tabular}{|c|c|c|c|c|c|c|c|c|c|c|c|}
\hline Sample & TR-89 & TR-89 & TR-89 & TR-89 & TR-89 & TR-89 & TR-01 & TR-01 & TR-01 & TR-01 & TR-30 \\
\hline Location & Core & Rim & Core & Rim & Core & Rim & Core & Rim & Core & Rim & Core \\
\hline $\mathrm{SiO}_{2}$ & 29.66 & 29.89 & 29.01 & 30.69 & 30.68 & 31.77 & 30.37 & 30.44 & 30.44 & 30.70 & 30.02 \\
\hline $\mathrm{TiO}_{2}$ & 35.54 & 36.43 & 35.20 & 35.31 & 35.42 & 32.82 & 36.41 & 37.24 & 36.48 & 34.47 & 39.37 \\
\hline $\mathrm{CaO}$ & 26.92 & 27.61 & 26.28 & 25.71 & 27.98 & 26.99 & 27.07 & 28.89 & 27.72 & 28.61 & 28.77 \\
\hline $\mathrm{Al}_{2} \mathrm{O}_{3}$ & 1.35 & 1.54 & 1.22 & 1.47 & 1.41 & 1.72 & 1.51 & 1.66 & 1.41 & 1.66 & 1.18 \\
\hline $\mathrm{Fe}_{2} \mathrm{O}_{3}$ & 2.68 & 1.75 & 1.96 & 1.78 & 1.70 & 2.15 & 1.68 & 1.41 & 1.62 & 1.41 & 0.84 \\
\hline $\mathrm{Na}_{2} \mathrm{O}$ & 0.05 & 0.03 & 0.05 & 0.27 & 0.05 & 0.17 & 0.00 & 0.00 & 0.06 & 0.05 & 0.04 \\
\hline $\mathrm{MgO}$ & 0.05 & 0.03 & 0.03 & 0.03 & 0.02 & 0.06 & 0.03 & 0.00 & 0.00 & 0.00 & 0.00 \\
\hline $\mathrm{V}_{2} \mathrm{O}_{3}$ & 0.31 & 0.41 & 0.29 & 0.47 & 0.38 & 0.33 & 0.39 & 0.39 & 0.33 & 0.30 & 0.38 \\
\hline $\mathrm{MnO}$ & 0.13 & 0.19 & 0.08 & 0.09 & 0.10 & 0.24 & 0.28 & 0.13 & 0.21 & 0.18 & 0.03 \\
\hline $\mathrm{BaO}$ & 0.00 & 0.09 & 0.18 & 0.17 & 0.12 & 0.21 & 0.18 & 0.18 & 0.16 & 0.23 & 0.15 \\
\hline $\mathrm{F}$ & 0.50 & 0.45 & 0.18 & 0.51 & 0.44 & 0.61 & 0.34 & 0.60 & 0.44 & 0.77 & 0.29 \\
\hline $\mathrm{H}_{2} \mathrm{O}^{* *}$ & 0.30 & 0.26 & 0.35 & 0.22 & 0.23 & 0.26 & 0.29 & 0.17 & 0.22 & 0.09 & 0.16 \\
\hline $\mathrm{OH} \equiv \mathrm{F}$ & 0.21 & 0.19 & 0.08 & 0.21 & 0.18 & 0.26 & 0.14 & 0.25 & 0.19 & 0.33 & 0.12 \\
\hline Total & 97.29 & 98.48 & 94.74 & 96.50 & 98.35 & 97.07 & 98.41 & 100.85 & 98.90 & 98.14 & 101.11 \\
\hline \multicolumn{12}{|c|}{ Cation proportions on the basis of 5 oxygens } \\
\hline $\mathrm{Si}$ & 1.004 & 0.998 & 1.007 & 1.038 & 1.025 & 1.071 & 1.013 & 0.994 & 1.011 & 1.028 & 0.976 \\
\hline $\mathrm{Ti}$ & 0.905 & 0.915 & 0.919 & 0.898 & 0.890 & 0.832 & 0.913 & 0.914 & 0.911 & 0.868 & 0.963 \\
\hline $\mathrm{Ca}$ & 0.976 & 0.988 & 0.977 & 0.932 & 1.001 & 0.975 & 0.967 & 1.010 & 0.987 & 1.027 & 1.002 \\
\hline $\mathrm{Al}$ & 0.054 & 0.061 & 0.050 & 0.059 & 0.056 & 0.068 & 0.059 & 0.064 & 0.055 & 0.066 & 0.045 \\
\hline $\mathrm{Fe}^{3+}$ & 0.068 & 0.044 & 0.051 & 0.045 & 0.043 & 0.055 & 0.042 & 0.035 & 0.040 & 0.036 & 0.021 \\
\hline $\mathrm{Na}$ & 0.003 & 0.002 & 0.003 & 0.018 & 0.003 & 0.011 & 0.000 & 0.000 & 0.004 & 0.003 & 0.002 \\
\hline $\mathrm{Mg}$ & 0.003 & 0.002 & 0.002 & 0.002 & 0.001 & 0.003 & 0.001 & 0.000 & 0.000 & 0.000 & 0.000 \\
\hline $\mathrm{V}$ & 0.009 & 0.011 & 0.008 & 0.013 & 0.010 & 0.009 & 0.010 & 0.010 & 0.009 & 0.008 & 0.010 \\
\hline $\mathrm{Mn}$ & 0.004 & 0.005 & 0.002 & 0.002 & 0.003 & 0.007 & 0.008 & 0.004 & 0.006 & 0.005 & 0.001 \\
\hline $\mathrm{Ba}$ & 0.000 & 0.001 & 0.002 & 0.002 & 0.002 & 0.003 & 0.002 & 0.002 & 0.002 & 0.003 & 0.002 \\
\hline Sum & 3.025 & 3.027 & 3.022 & 3.008 & 3.033 & 3.033 & 3.016 & 3.033 & 3.026 & 3.043 & 3.022 \\
\hline $\mathrm{F}$ & 0.053 & 0.048 & 0.020 & 0.054 & 0.046 & 0.065 & 0.036 & 0.062 & 0.046 & 0.082 & 0.030 \\
\hline $\mathrm{Al}+\mathrm{Fe}^{3+}$ & 0.122 & 0.105 & 0.101 & 0.104 & 0.098 & 0.123 & 0.101 & 0.098 & 0.096 & 0.101 & 0.066 \\
\hline $\mathrm{Ti}^{4+} /\left(\mathrm{Al}+\mathrm{Fe}^{3+}\right)$ & 7.422 & 8.737 & 9.103 & 8.654 & 9.038 & 6.767 & 9.007 & 9.283 & 9.521 & 8.576 & 14.633 \\
\hline $\mathrm{OH}^{* *}$ & 0.068 & 0.057 & 0.081 & 0.050 & 0.052 & 0.058 & 0.065 & 0.036 & 0.049 & 0.019 & 0.036 \\
\hline $\mathrm{X}_{\mathrm{F}} \mathrm{g}$ & 0.44 & 0.45 & 0.20 & 0.52 & 0.47 & 0.53 & 0.36 & 0.63 & 0.49 & 0.81 & 0.46 \\
\hline
\end{tabular}

$\mathrm{XF}: \mathrm{F} /(\mathrm{F}+\mathrm{OH})$; $\mathrm{OH}$ calculated as $\mathrm{OH}=(\mathrm{Al}+\mathrm{Fe} 3+)-\mathrm{F}^{* *}$ calculated.

Table 4A. Microprobe analyses for biotite of the Major Isidoro granites.

\begin{tabular}{|c|c|c|c|c|c|c|c|c|c|c|c|c|c|c|}
\hline Sample & $\begin{array}{c}\text { TR- } \\
13\end{array}$ & $\begin{array}{c}\text { TR- } \\
13\end{array}$ & $\begin{array}{c}\text { TR- } \\
13\end{array}$ & $\begin{array}{c}\text { TR- } \\
13\end{array}$ & $\begin{array}{c}\text { TR- } \\
13\end{array}$ & $\begin{array}{c}\text { TR- } \\
13\end{array}$ & $\begin{array}{c}\text { TR- } \\
13\end{array}$ & $\begin{array}{c}\text { TR- } \\
13\end{array}$ & $\begin{array}{c}\text { TR- } \\
22\end{array}$ & $\begin{array}{c}\text { TR- } \\
22\end{array}$ & $\begin{array}{c}\text { TR- } \\
22\end{array}$ & $\begin{array}{c}\text { TR- } \\
22\end{array}$ & $\begin{array}{c}\text { TR- } \\
22\end{array}$ & $\begin{array}{c}\text { TR- } \\
22\end{array}$ \\
\hline Location & Core & Rim & Core & Rim & Core & Rim & Core & Rim & Core & Rim & Core & Rim & Core & Rim \\
\hline $\mathrm{SiO}_{2}$ & 36.42 & 36.53 & 36.66 & 36.38 & 37.03 & 36.93 & 36.79 & 36.60 & 36.62 & 36.92 & 36.79 & 36.32 & 35.96 & 36.29 \\
\hline $\mathrm{Al}_{2} \mathrm{O}_{3}$ & 16.36 & 15.45 & 15.60 & 15.26 & 16.03 & 16.54 & 16.13 & 16.09 & 15.06 & 14.81 & 15.06 & 15.02 & 14.92 & 14.65 \\
\hline $\mathrm{FeO}$ & 20.63 & 21.05 & 21.11 & 21.00 & 20.90 & 19.82 & 21.26 & 20.74 & 20.70 & 20.61 & 21.41 & 21.23 & 21.17 & 20.52 \\
\hline $\mathrm{MgO}$ & 9.19 & 9.72 & 9.08 & 9.57 & 9.75 & 10.03 & 8.69 & 8.57 & 10.54 & 10.59 & 10.29 & 10.30 & 10.88 & 11.28 \\
\hline $\mathrm{TiO}_{2}$ & 3.36 & 3.24 & 3.11 & 3.29 & 2.95 & 3.15 & 2.91 & 3.80 & 2.50 & 2.53 & 2.29 & 2.05 & 1.84 & 1.97 \\
\hline $\mathrm{MnO}$ & 0.27 & 0.32 & 0.39 & 0.29 & 0.27 & 0.30 & 0.48 & 0.43 & 0.46 & 0.37 & 0.57 & 0.37 & 0.46 & 0.50 \\
\hline $\mathrm{Cr}_{2} \mathrm{O}_{3}$ & bdl & bdl & 0.04 & bdl & bdl & bdl & bdl & bdl & bdl & bdl & bdl & 0.12 & bdl & 0.06 \\
\hline $\mathrm{V}_{2} \mathrm{O}_{3}$ & 0.05 & 0.04 & 0.09 & 0.11 & 0.07 & 0.04 & 0.04 & 0.09 & 0.03 & 0.02 & 0.10 & 0.06 & 0.04 & 0.07 \\
\hline $\mathrm{K}_{2} \mathrm{O}$ & 9.76 & 9.59 & 9.65 & 9.63 & 9.89 & 9.76 & 9.72 & 9.87 & 9.66 & 9.73 & 9.61 & 9.70 & 9.57 & 9.72 \\
\hline
\end{tabular}


Table 4A. Continuation.

\begin{tabular}{|c|c|c|c|c|c|c|c|c|c|c|c|c|c|c|}
\hline Sample & $\begin{array}{c}\text { TR- } \\
13\end{array}$ & $\begin{array}{c}\text { TR- } \\
13\end{array}$ & $\begin{array}{c}\text { TR- } \\
13\end{array}$ & $\begin{array}{c}\text { TR- } \\
13\end{array}$ & $\begin{array}{c}\text { TR- } \\
13\end{array}$ & $\begin{array}{c}\text { TR- } \\
13\end{array}$ & $\begin{array}{c}\text { TR- } \\
13\end{array}$ & $\begin{array}{c}\text { TR- } \\
13\end{array}$ & $\begin{array}{c}\text { TR- } \\
22\end{array}$ & $\begin{array}{c}\text { TR- } \\
22\end{array}$ & $\begin{array}{c}\text { TR- } \\
22\end{array}$ & $\begin{array}{c}\text { TR- } \\
22\end{array}$ & $\begin{array}{c}\text { TR- } \\
22\end{array}$ & $\begin{array}{c}\text { TR- } \\
22\end{array}$ \\
\hline Location & Core & Rim & Core & Rim & Core & Rim & Core & Rim & Core & Rim & Core & Rim & Core & Rim \\
\hline $\mathrm{Na}_{2} \mathrm{O}$ & 0.09 & 0.11 & 0.10 & 0.06 & 0.08 & 0.12 & 0.09 & 0.09 & 0.08 & 0.13 & 0.12 & 0.14 & 0.08 & 0.10 \\
\hline $\mathrm{CaO}$ & 0.05 & 0.06 & 0.03 & 0.07 & 0.04 & 0.03 & 0.01 & 0.04 & 0.03 & 0.08 & 0.02 & 0.02 & 0.03 & 0.04 \\
\hline $\mathrm{BaO}$ & 0.06 & 0.06 & bdl & 0.14 & 0.15 & 0.12 & 0.14 & bdl & 0.10 & 0.23 & 0.10 & 0.20 & 0.14 & 0.06 \\
\hline F & 0.60 & 0.83 & 0.86 & 0.68 & 0.88 & 0.81 & 0.68 & 0.60 & 0.54 & 0.72 & 0.63 & 0.54 & 0.55 & 0.62 \\
\hline $\mathrm{Cl}$ & 0.05 & 0.03 & 0.06 & 0.06 & 0.04 & 0.06 & 0.06 & 0.09 & 0.05 & 0.06 & 0.04 & 0.06 & 0.07 & 0.02 \\
\hline $\mathrm{O}=(\mathrm{F}, \mathrm{Cl})$ & 0.26 & 0.35 & 0.38 & 0.30 & 0.38 & 0.35 & 0.30 & 0.27 & 0.24 & 0.32 & 0.28 & 0.24 & 0.25 & 0.26 \\
\hline Total & 96.59 & 96.65 & 96.41 & 96.23 & 97.70 & 97.35 & 96.69 & 96.74 & 96.13 & 96.49 & 96.75 & 95.87 & 95.45 & 95.63 \\
\hline \multicolumn{15}{|c|}{ Cation proportions on the basis of 22 oxygens } \\
\hline $\mathrm{Si}$ & 5.548 & 5.580 & 5.619 & 5.588 & 5.593 & 5.559 & 5.619 & 5.574 & 5.616 & 5.651 & 5.625 & 5.614 & 5.583 & 5.607 \\
\hline $\mathrm{Al}^{\mathrm{IV}}$ & 2.452 & 2.420 & 2.381 & 2.412 & 2.407 & 2.441 & 2.381 & 2.426 & 2.384 & 2.349 & 2.375 & 2.386 & 2.417 & 2.393 \\
\hline $\mathrm{Z}$ site & 8.000 & 8.000 & 8.000 & 8.000 & 8.000 & 8.000 & 8.000 & 8.000 & 8.000 & 8.000 & 8.000 & 8.000 & 8.000 & 8.000 \\
\hline $\mathrm{Al}^{\mathrm{VI}}$ & 0.484 & 0.362 & 0.437 & 0.351 & 0.447 & 0.493 & 0.523 & 0.462 & 0.338 & 0.323 & 0.339 & 0.350 & 0.314 & 0.274 \\
\hline $\mathrm{Fe}$ & 2.628 & 2.689 & 2.706 & 2.697 & 2.639 & 2.495 & 2.715 & 2.641 & 2.655 & 2.637 & 2.738 & 2.744 & 2.749 & 2.651 \\
\hline $\mathrm{Mg}$ & 2.086 & 2.213 & 2.075 & 2.190 & 2.195 & 2.252 & 1.977 & 1.946 & 2.410 & 2.416 & 2.346 & 2.374 & 2.519 & 2.598 \\
\hline $\mathrm{Ti}$ & 0.385 & 0.372 & 0.359 & 0.380 & 0.335 & 0.356 & 0.334 & 0.436 & 0.288 & 0.291 & 0.263 & 0.238 & 0.214 & 0.229 \\
\hline $\mathrm{Mn}$ & 0.034 & 0.041 & 0.050 & 0.038 & 0.034 & 0.038 & 0.061 & 0.055 & 0.059 & 0.048 & 0.073 & 0.048 & 0.061 & 0.066 \\
\hline $\mathrm{Cr}$ & - & - & 0.004 & - & - & - & - & - & - & - & - & 0.014 & - & 0.007 \\
\hline $\mathrm{V}$ & 0.006 & 0.005 & 0.011 & 0.014 & 0.009 & 0.005 & 0.005 & 0.011 & 0.004 & 0.003 & 0.012 & 0.007 & 0.004 & 0.009 \\
\hline Y site & 5.623 & 5.681 & 5.642 & 5.670 & 5.659 & 5.640 & 5.616 & 5.551 & 5.754 & 5.718 & 5.771 & 5.776 & 5.862 & 5.833 \\
\hline $\mathrm{K}$ & 1.896 & 1.869 & 1.887 & 1.888 & 1.907 & 1.875 & 1.894 & 1.918 & 1.891 & 1.899 & 1.875 & 1.913 & 1.895 & 1.916 \\
\hline $\mathrm{Na}$ & 0.028 & 0.031 & 0.028 & 0.018 & 0.024 & 0.036 & 0.025 & 0.027 & 0.023 & 0.039 & 0.036 & 0.041 & 0.024 & 0.028 \\
\hline $\mathrm{Ca}$ & 0.008 & 0.010 & 0.006 & 0.011 & 0.007 & 0.005 & 0.002 & 0.007 & 0.004 & 0.013 & 0.003 & 0.004 & 0.005 & 0.006 \\
\hline $\mathrm{Ba}$ & 0.003 & 0.003 & - & 0.008 & 0.009 & 0.007 & 0.008 & - & 0.006 & 0.014 & 0.006 & 0.012 & 0.009 & 0.004 \\
\hline $\mathrm{X}$ site & 1.935 & 1.913 & 1.921 & 1.925 & 1.946 & 1.923 & 1.930 & 1.952 & 1.924 & 1.966 & 1.920 & 1.971 & 1.933 & 1.954 \\
\hline $\mathrm{F}$ & 0.289 & 0.400 & 0.419 & 0.330 & 0.422 & 0.385 & 0.330 & 0.287 & 0.260 & 0.348 & 0.306 & 0.263 & 0.271 & 0.301 \\
\hline $\mathrm{Cl}$ & 0.013 & 0.006 & 0.015 & 0.015 & 0.011 & 0.014 & 0.015 & 0.022 & 0.013 & 0.016 & 0.011 & 0.015 & 0.017 & 0.005 \\
\hline $\begin{array}{l}\mathrm{Fe} / \\
(\mathrm{Fe}+\mathrm{Mg})\end{array}$ & 0.557 & 0.549 & 0.566 & 0.552 & 0.546 & 0.526 & 0.579 & 0.576 & 0.524 & 0.522 & 0.538 & 0.536 & 0.522 & 0.505 \\
\hline $\mathrm{FeO} / \mathrm{MgO}$ & 2.245 & 2.166 & 2.324 & 2.195 & 2.143 & 1.975 & 2.447 & 2.419 & 1.964 & 1.946 & 2.080 & 2.060 & 1.945 & 1.820 \\
\hline $\mathrm{Al}^{\mathrm{Tot}}$ & 2.937 & 2.782 & 2.818 & 2.762 & 2.853 & 2.934 & 2.903 & 2.888 & 2.722 & 2.672 & 2.713 & 2.736 & 2.730 & 2.667 \\
\hline
\end{tabular}

bdl: below detection limit.

Table 4B. Microprobe analyses for biotite of the Monteirópolis granites.

\begin{tabular}{lcccccccccccc}
\hline Sample & TR-89 & TR-89 & TR-89 & TR-89 & TR-89 & TR-89 & TR-89 & TR-89 & TR-01 & TR-01 & TR-01 & TR-01 \\
\hline Location & Core & Rim & Core & Rim & Core & Rim & Core & Rim & Core & Rim & Core & Rim \\
\hline $\mathrm{SiO}_{2}$ & 38.274 & 37.954 & 38.601 & 35.452 & 37.703 & 38.159 & 38.294 & 38.223 & 37.107 & 36.967 & 36.246 & 37.111 \\
$\mathrm{Al}_{2} \mathrm{O}_{3}$ & 14.242 & 14.417 & 14.887 & 14.22 & 14.24 & 14.159 & 14.461 & 13.868 & 15.061 & 14.549 & 15.876 & 15.401 \\
$\mathrm{FeO}$ & 17.715 & 17.674 & 17.745 & 21.489 & 18.562 & 18.763 & 18.392 & 17.702 & 20.186 & 20.127 & 20.966 & 19.994 \\
$\mathrm{MgO}$ & 12.693 & 12.56 & 12.412 & 11.47 & 12.304 & 12.928 & 12.54 & 13.206 & 9.931 & 10.29 & 9.685 & 10.344 \\
$\mathrm{TiO}_{2}$ & 1.631 & 1.312 & 2.13 & 1.495 & 1.23 & 1.112 & 1.728 & 1.46 & 2.721 & 2.779 & 3.059 & 2.836 \\
$\mathrm{MnO}$ & 0.625 & 0.512 & 0.373 & 0.478 & 0.44 & 0.457 & 0.416 & 0.357 & 0.507 & 0.413 & 0.47 & 0.619 \\
$\mathrm{Cr}_{2} \mathrm{O}_{3}$ & 0.069 & 0.13 & 0.104 & $b d l$ & $b d l$ & $b d l$ & $b d l$ & $b d l$ & $b d l$ & 0.115 & 0.079 & bdl \\
$\mathrm{V}_{2} \mathrm{O}_{3}$ & 0.065 & 0.043 & 0.073 & 0.057 & 0.137 & 0.011 & 0.063 & 0.046 & 0.034 & 0.085 & 0.076 & 0.061 \\
$\mathrm{~K}_{2} \mathrm{O}$ & 9.747 & 9.761 & 9.943 & 9.328 & 9.783 & 9.708 & 9.69 & 9.653 & 9.633 & 9.719 & 9.972 & 9.586 \\
$\mathrm{Na}_{2} \mathrm{O}$ & 0.043 & 0.086 & 0.032 & 0.119 & 0.042 & 0.037 & 0.066 & 0.115 & 0.123 & 0.075 & 0.16 & 0.123 \\
$\mathrm{CaO}$ & 0.048 & 0.044 & 0.071 & 0.084 & 0.053 & 0.031 & 0.091 & 0.13 & 0.045 & bdl & 0.018 & 0.047 \\
\hline & & & & & & & & & & & & Continue...
\end{tabular}


Table 4B. Continuation.

\begin{tabular}{|c|c|c|c|c|c|c|c|c|c|c|c|c|}
\hline Sample & TR-89 & TR-89 & TR-89 & TR-89 & TR-89 & TR-89 & TR-89 & TR-89 & TR-01 & TR-01 & TR-01 & TR-01 \\
\hline Location & Core & Rim & Core & Rim & Core & Rim & Core & Rim & Core & Rim & Core & Rim \\
\hline $\mathrm{BaO}$ & 0.182 & bdl & 0.209 & 0.039 & 0.139 & 0.198 & bdl & 0.133 & 0.19 & 0.116 & 0.099 & 0.154 \\
\hline $\mathrm{F}$ & 1.193 & 1.182 & 1.135 & 1.062 & 1.118 & 1.243 & 1.193 & 1.288 & 0.675 & 0.749 & 0.601 & 0.8 \\
\hline $\mathrm{Cl}$ & bdl & 0.013 & bdl & bdl & bdl & bdl & 0.003 & 0.024 & 0.037 & 0.024 & 0.028 & 0.018 \\
\hline $\mathrm{O}=(\mathrm{F}, \mathrm{Cl})$ & 0.502 & 0.501 & 0.478 & 0.447 & 0.471 & 0.523 & 0.503 & 0.548 & 0.293 & 0.321 & 0.259 & 0.341 \\
\hline Total & 96.02 & 95.19 & 97.24 & 94.85 & 95.28 & 96.28 & 96.43 & 95.66 & 95.96 & 95.69 & 97.08 & 96.75 \\
\hline \multicolumn{13}{|c|}{ Cation proportions on the basis of 22 oxygens } \\
\hline $\mathrm{Si}$ & 5.804 & 5.801 & 5.771 & 5.570 & 5.791 & 5.799 & 5.786 & 5.819 & 5.688 & 5.690 & 5.526 & 5.638 \\
\hline $\mathrm{Al}^{\mathrm{IV}}$ & 2.196 & 2.199 & 2.229 & 2.430 & 2.209 & 2.201 & 2.214 & 2.181 & 2.312 & 2.310 & 2.474 & 2.362 \\
\hline $\mathrm{Z}$ site & 8.000 & 8.000 & 8.000 & 8.000 & 8.000 & 8.000 & 8.000 & 8.000 & 8.000 & 8.000 & 8.000 & 8.000 \\
\hline $\mathrm{Al}^{\mathrm{VI}}$ & 0.350 & 0.398 & 0.395 & 0.203 & 0.369 & 0.336 & 0.361 & 0.308 & 0.409 & 0.329 & 0.379 & 0.395 \\
\hline $\mathrm{Fe}$ & 2.247 & 2.259 & 2.219 & 2.824 & 2.384 & 2.385 & 2.324 & 2.254 & 2.588 & 2.591 & 2.673 & 2.540 \\
\hline $\mathrm{Mg}$ & 2.870 & 2.862 & 2.766 & 2.687 & 2.817 & 2.929 & 2.825 & 2.997 & 2.269 & 2.361 & 2.201 & 2.343 \\
\hline $\mathrm{Ti}$ & 0.186 & 0.151 & 0.239 & 0.177 & 0.142 & 0.127 & 0.196 & 0.167 & 0.314 & 0.322 & 0.351 & 0.324 \\
\hline $\mathrm{M}$ & 0.080 & 0.066 & 0.047 & 0.064 & 0.057 & 0.059 & 0.053 & 0.046 & 0.066 & 0.054 & 0.061 & 0.080 \\
\hline $\mathrm{Cr}$ & 0.008 & 0.016 & 0.012 & - & - & - & - & - & - & 0.014 & 0.010 & - \\
\hline $\mathrm{V}$ & 0.008 & 0.005 & 0.009 & 0.007 & 0.017 & 0.001 & 0.008 & 0.006 & 0.004 & 0.010 & 0.009 & 0.007 \\
\hline Y site & 5.749 & 5.758 & 5.687 & 5.961 & 5.787 & 5.837 & 5.767 & 5.778 & 5.650 & 5.680 & 5.684 & 5.689 \\
\hline $\mathrm{K}$ & 1.886 & 1.903 & 1.897 & 1.870 & 1.917 & 1.882 & 1.868 & 1.875 & 1.884 & 1.908 & 1.940 & 1.858 \\
\hline $\mathrm{Na}$ & 0.013 & 0.025 & 0.009 & 0.036 & 0.013 & 0.011 & 0.019 & 0.034 & 0.037 & 0.022 & 0.047 & 0.036 \\
\hline $\mathrm{Ca}$ & 0.008 & 0.007 & 0.011 & 0.014 & 0.009 & 0.005 & 0.015 & 0.021 & 0.007 & - & 0.003 & 0.008 \\
\hline $\mathrm{Ba}$ & 0.011 & - & 0.012 & 0.002 & 0.008 & 0.012 & - & 0.008 & 0.011 & 0.007 & 0.006 & 0.009 \\
\hline $\mathrm{X}$ site & 1.917 & 1.936 & 1.929 & 1.922 & 1.947 & 1.910 & 1.902 & 1.938 & 1.939 & 1.938 & 1.996 & 1.911 \\
\hline $\mathrm{F}$ & 0.572 & 0.571 & 0.537 & 0.528 & 0.543 & 0.597 & 0.570 & 0.620 & 0.327 & 0.365 & 0.290 & 0.384 \\
\hline $\mathrm{Cl}$ & - & 0.003 & - & - & - & - & 0.001 & 0.006 & 0.010 & 0.006 & 0.007 & 0.005 \\
\hline $\begin{array}{l}\mathrm{Fe} / \\
(\mathrm{Fe}+\mathrm{Mg})\end{array}$ & 0.439 & 0.441 & 0.445 & 0.512 & 0.458 & 0.449 & 0.451 & 0.429 & 0.533 & 0.523 & 0.548 & 0.520 \\
\hline $\mathrm{FeO} / \mathrm{MgO}$ & 1.396 & 1.407 & 1.430 & 1.873 & 1.509 & 1.451 & 1.467 & 1.340 & 2.033 & 1.956 & 2.165 & 1.933 \\
\hline $\mathrm{Al}^{\mathrm{Tot}}$ & 2.546 & 2.597 & 2.623 & 2.633 & 2.578 & 2.536 & 2.575 & 2.488 & 2.721 & 2.639 & 2.853 & 2.757 \\
\hline
\end{tabular}

bdl: below detection limit.

Table 5. Microprobe analyses, cation proportions, and pistacite contents for epidote of the studied plutons.

\begin{tabular}{|c|c|c|c|c|c|c|c|c|c|c|c|}
\hline Sample & TR-89 & TR-89 & TR-89 & TR-89 & TR-89 & TR-22 & TR-22 & TR-22 & TR-22 & TR-22 & TR-22 \\
\hline Location & Core & Rim & Core & Rim & Rim & Core & Rim & Core & Rim & Core & Rim \\
\hline $\mathrm{SiO}_{2}$ & 38.213 & 37.667 & 37.938 & 38.328 & 36.143 & 36.531 & 37.717 & 37.741 & 37.687 & 37.88 & 36.206 \\
\hline $\mathrm{CaO}$ & 23.366 & 23.448 & 23.21 & 23.143 & 23.267 & 23.428 & 23.646 & 23.442 & 23.598 & 23.421 & 23.14 \\
\hline $\mathrm{Al}_{2} \mathrm{O}_{3}$ & 21.634 & 22.018 & 21.456 & 22.502 & 22.2 & 21.968 & 22.612 & 22.376 & 22.798 & 22.555 & 22.81 \\
\hline $\mathrm{Fe}_{2} \mathrm{O}_{3}$ & 15.481 & 15.231 & 15.020 & 14.273 & 15.055 & 13.923 & 13.896 & 14.330 & 13.419 & 13.878 & 14.507 \\
\hline $\mathrm{SrO}$ & 0.32 & 0.456 & 0.443 & 0.424 & 0.193 & 0.149 & 0.143 & 0.096 & 0.154 & 0.1 & 0.059 \\
\hline $\mathrm{MnO}$ & 0.186 & 0.198 & 0.242 & 0.106 & 0.268 & 0.423 & 0.367 & 0.412 & 0.425 & 0.261 & 0.435 \\
\hline $\mathrm{TiO}_{2}$ & 0.152 & bdl & 0.009 & 0.06 & 0.122 & 0.07 & 0.104 & 0.188 & 0.296 & 0.162 & 0.005 \\
\hline $\mathrm{Na}_{2} \mathrm{O}$ & 0.017 & 0.031 & 0.049 & bdl & 0.047 & 0.041 & bdl & 0.016 & bdl & 0.016 & 0.024 \\
\hline $\mathrm{MgO}$ & 0.005 & 0.037 & 0.019 & 0.011 & 0.049 & 0.084 & bdl & 0.044 & 0.011 & bdl & 0.003 \\
\hline $\mathrm{K}_{2} \mathrm{O}$ & bdl & bdl & 0.018 & 0.005 & bdl & 0.017 & 0.003 & bdl & bdl & bdl & bdl \\
\hline $\mathrm{Cr}_{2} \mathrm{O}_{3}$ & bdl & bdl & 0.107 & 0.071 & 0.059 & 0.071 & bdl & 0.06 & bdl & bdl & 0.025 \\
\hline $\mathrm{NiO}$ & 0.007 & 0.087 & bdl & 0.041 & 0.031 & 0.066 & 0.026 & bdl & bdl & bdl & 0.059 \\
\hline $\mathrm{BaO}$ & bdl & 0.023 & 0.133 & 0.041 & 0.075 & 0.05 & 0.017 & bdl & 0.017 & bdl & 0.04 \\
\hline $\mathrm{V}_{2} \mathrm{O}_{3}$ & 0.067 & 0.069 & 0.107 & 0.051 & 0.044 & 0.037 & 0.055 & 0.054 & 0.054 & 0.101 & 0.069 \\
\hline Total & 99.448 & 99.265 & 98.751 & 99.056 & 97.553 & 96.858 & 98.586 & 98.759 & 98.459 & 98.374 & 97.382 \\
\hline
\end{tabular}


Braz. J. Geol. (2020), 50(2): e20190110

Table 5. Continuation.

\begin{tabular}{|c|c|c|c|c|c|c|c|c|c|c|c|}
\hline Sample & TR-89 & TR-89 & TR-89 & TR-89 & TR-89 & TR-22 & TR-22 & TR-22 & TR-22 & TR-22 & TR-22 \\
\hline Location & Core & Rim & Core & Rim & Rim & Core & Rim & Core & Rim & Core & Rim \\
\hline \multicolumn{12}{|c|}{ Cation proportions on the basis of 12.5 oxygens } \\
\hline $\mathrm{Si}$ & 3.026 & 2.994 & 3.030 & 3.033 & 2.928 & 2.972 & 3.002 & 3.000 & 2.999 & 3.015 & 2.929 \\
\hline $\mathrm{Ca}$ & 1.982 & 1.997 & 1.986 & 1.962 & 2.020 & 2.042 & 2.016 & 1.997 & 2.012 & 1.997 & 2.006 \\
\hline $\mathrm{Al}$ & 2.019 & 2.063 & 2.020 & 2.098 & 2.120 & 2.106 & 2.121 & 2.097 & 2.138 & 2.116 & 2.175 \\
\hline $\mathrm{Fe}$ & 0.922 & 0.911 & 0.903 & 0.850 & 0.918 & 0.852 & 0.832 & 0.857 & 0.804 & 0.831 & 0.883 \\
\hline Sr & 0.015 & 0.021 & 0.021 & 0.019 & 0.009 & 0.007 & 0.007 & 0.004 & 0.007 & 0.005 & 0.003 \\
\hline $\mathrm{Mn}$ & 0.012 & 0.013 & 0.016 & 0.007 & 0.018 & 0.029 & 0.025 & 0.028 & 0.029 & 0.018 & 0.030 \\
\hline $\mathrm{Ti}$ & 0.009 & 0.000 & 0.001 & 0.004 & 0.007 & 0.004 & 0.006 & 0.011 & 0.018 & 0.010 & 0.000 \\
\hline $\mathrm{Na}$ & 0.003 & 0.005 & 0.008 & 0.000 & 0.007 & 0.006 & 0.000 & 0.002 & 0.000 & 0.002 & 0.004 \\
\hline $\mathrm{Mg}$ & 0.001 & 0.004 & 0.002 & 0.001 & 0.006 & 0.010 & 0.000 & 0.005 & 0.001 & 0.000 & 0.000 \\
\hline K & 0.000 & 0.000 & 0.002 & 0.001 & 0.000 & 0.002 & 0.000 & 0.000 & 0.000 & 0.000 & 0.000 \\
\hline $\mathrm{Cr}$ & 0.000 & 0.000 & 0.007 & 0.004 & 0.004 & 0.005 & 0.000 & 0.004 & 0.000 & 0.000 & 0.002 \\
\hline $\mathrm{Ni}$ & 0.000 & 0.006 & 0.000 & 0.003 & 0.002 & 0.004 & 0.002 & 0.000 & 0.000 & 0.000 & 0.004 \\
\hline $\mathrm{Ba}$ & 0.000 & 0.001 & 0.004 & 0.001 & 0.002 & 0.002 & 0.001 & 0.000 & 0.001 & 0.000 & 0.001 \\
\hline $\mathrm{V}$ & 0.004 & 0.004 & 0.007 & 0.003 & 0.003 & 0.002 & 0.004 & 0.003 & 0.003 & 0.006 & 0.004 \\
\hline Total & 7.994 & 8.019 & 8.006 & 7.986 & 8.045 & 8.045 & 8.014 & 8.009 & 8.011 & 8.000 & 8.041 \\
\hline $\begin{array}{l}\mathrm{Ps}=100 \mathrm{x} \\
\mathrm{Fe} /(\mathrm{Fe}+\mathrm{Al})\end{array}$ & 31 & 31 & 31 & 29 & 30 & 29 & 28 & 29 & 27 & 28 & 29 \\
\hline
\end{tabular}

bdl: below detection limit.

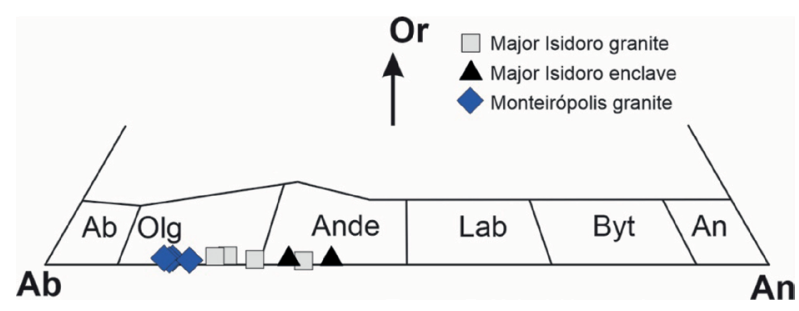

Ab: Albite; Olig: Oligoclase; Ande: Andesine; Lab: Labradorite; Byt: Bytownite; An: Anorthite.

Figure 3. Ternary molecular Albite-Anortite-Orthoclase feldspar diagram showing the compositional variations of plagioclase from the studied granites.

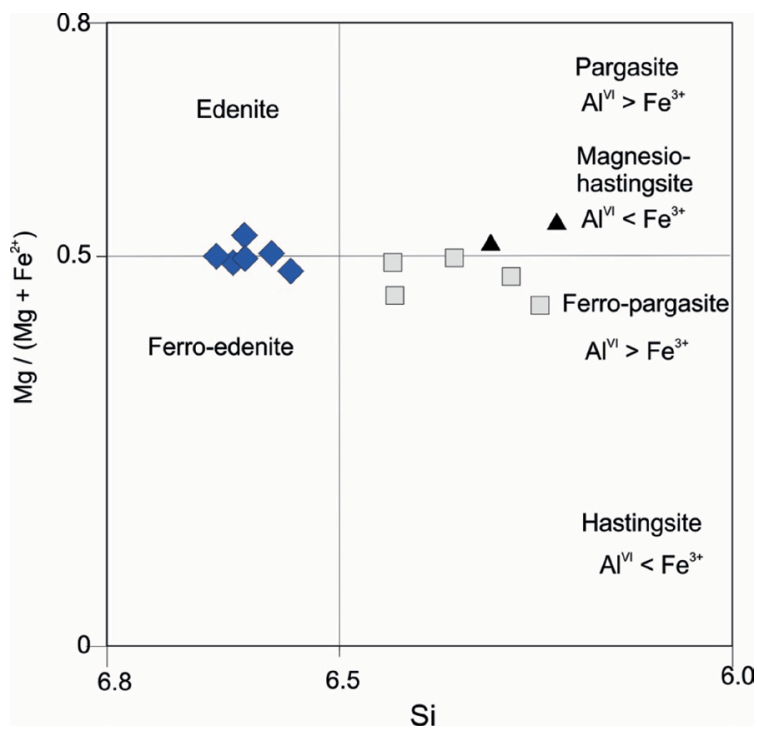

Figure 4. Classification diagram of amphibole following Leake et al. (1997). Symbols as in Figure 3.

\section{DISCUSSION}

\section{Intensive crystallization parameters}

\section{Near-solidus temperature}

Blundy and Holland (1990) proposed a hornblende-plagioclase thermometer with estimated uncertainties of $\pm 75^{\circ} \mathrm{C}$, in a temperature range of $500-1,100^{\circ} \mathrm{C}$, for quartz-saturated rocks. In a later work, Holland and Blundy (1994) extended the formulation of Blundy and Holland (1990) to embrace a broad range of bulk compositions (e.g., natural garnet amphibolites). The calibration based on the equilibrium: edenite + albite $=$ richterite + anorthite $($ reaction $\mathrm{B}$ of Holland and Blundy 1994) shows lower values of temperature than those estimated using reaction A of Holland and Blundy (1994), and are considered to be the most reliable because they more precisely reproduce the $\mathrm{T}$ estimated by other independent methods such as garnet-hornblende and clinopyroxene-hornblende (Anderson 1996, Bachmann and Dungan 2002). This thermometer works well in the ranges of $400-1,000^{\circ} \mathrm{C}\left( \pm 35-40^{\circ} \mathrm{C}\right)$ and 1 to $15 \mathrm{kbar}$ over a broad range of bulk compositions. Molina et al. (2015) calibrated an empirical amphibole/liquid Mg partitioning thermometer using regression methods and observed that their T estimates are consistent with those of the edenite-albite-richterite-anorthite thermometer of Holland and Blundy (1994). Near-solidus hornblende-plagioclase temperatures (reaction B of Holland and Blundy 1994) for the Major Isidoro and Monteirópolis plutons range from 680 to 720 and from 660 to $700^{\circ} \mathrm{C}$, respectively. 

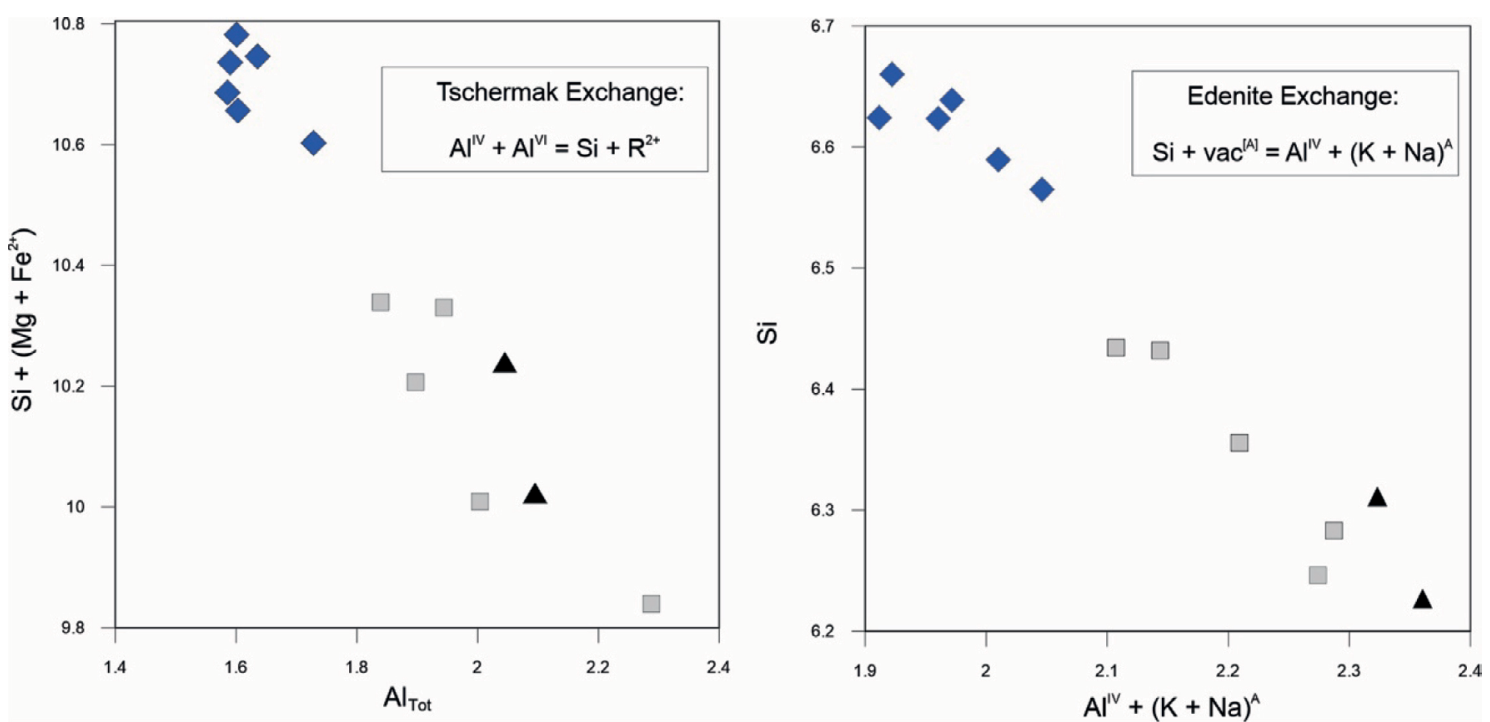

Figure 5. Compositional variations of amphibole from the studied granites. (A) Tschermak and (B) Edenite substitutions. Symbols as in Figure 3.

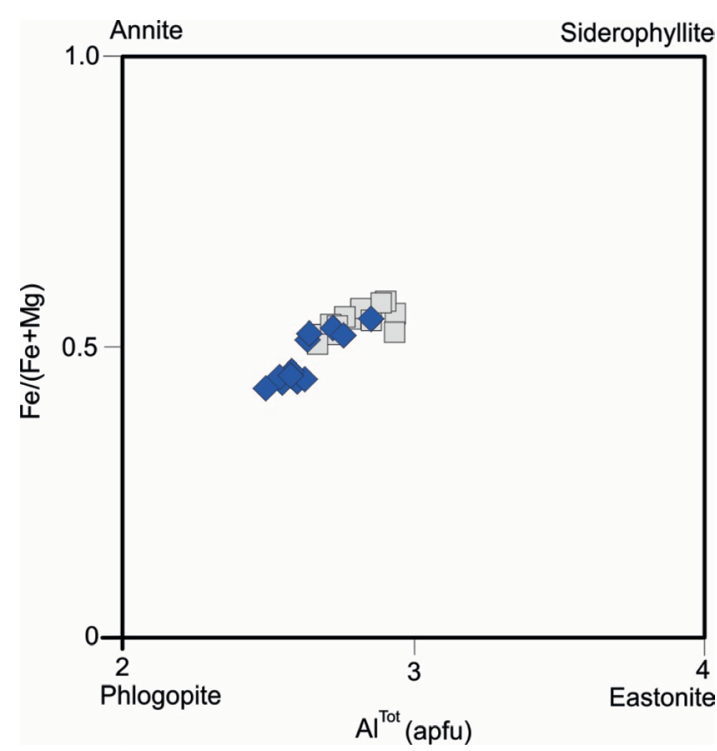

Figure 6. Compositional variations of biotite from the studied granites in the $\mathrm{Fe} /(\mathrm{Fe}+\mathrm{Mg})$ vs. Al ${ }^{\mathrm{Tot}}$ diagram. Symbols as in Figure 3.

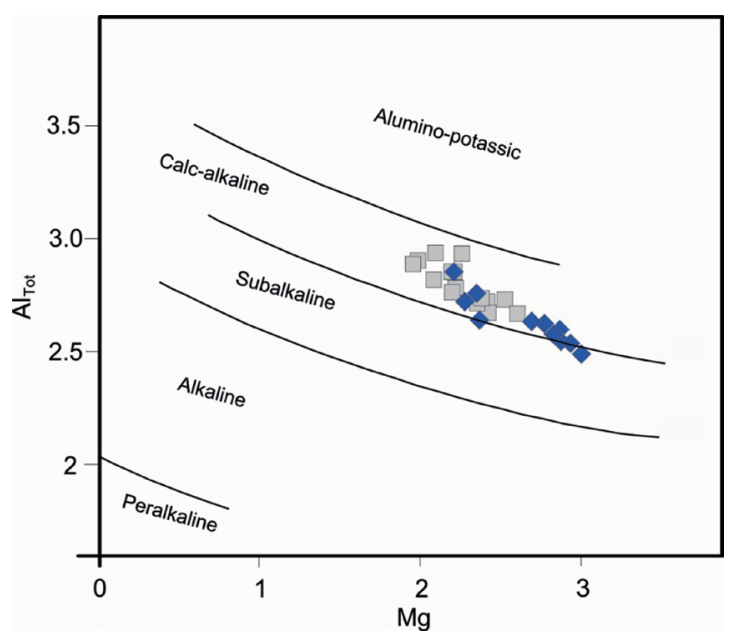

Figure 7. Compositional variations of biotites in the $\mathrm{Al}_{\text {Tot }} v$ s. $\mathrm{Mg}$ diagram. Symbols as in Figure 3.

\section{Near-liquidus temperature}

Watson and Harrison (1983) developed a solubility model based on the relationship between zircon crystallization and melt composition, and defined a saturation behavior of $\mathrm{Zr}$ as a function of temperature and magma composition. This model could be used when zircon was one of the earliest minerals to crystallize and assumes that zircon was not a cumulate phase, xenocrystic, or inherited from the source region. Sensitivity tests reinforce previous thoughts (see Watson \& Harrison 1983) and indicate that temperature and composition are the two dominant controls on zircon solubility in crustal melts with no observable effects due to pressure (up to $25 \mathrm{kbar}$ ) or variable water content (Boehnke et al. 2013). Estimates of the near liquidus temperature can be obtained by the Equation 1, using whole-rock analyses:

$\mathrm{T}_{\mathrm{Zr}}\left({ }^{\circ} \mathrm{C}\right)=\{(12,900) /[\ln (497,644 / \mathrm{Zr})+3.8+$
$0.85(\mathrm{M}-1)]-273.15\}$

According to this method, the calculated temperatures for the Major Isidoro pluton vary from 740 to $868^{\circ} \mathrm{C}$ (avg. $818^{\circ} \mathrm{C}$ ) and to the Monteirópolis pluton temperature ranges from 763 to $856^{\circ} \mathrm{C}$ (avg. $805^{\circ} \mathrm{C}$ ). Very low $\mathrm{Zr}$ saturation temperatures for some samples seem to underestimate the liquidus temperature with values slightly higher than those obtained to near-solidus temperature. The studied plutons present zircon cores inherited from the source, which interfere in the obtained $\mathrm{Zr}$ saturation temperatures (mean $\mathrm{T}_{\mathrm{Zr}}$ for inheritance-rich granites of $766^{\circ} \mathrm{C}$ were found by Miller et al. 2003). Green and Watson (1982) and Harrison and Watson (1984) related $\mathrm{P}_{2} \mathrm{O}_{5}$ contents at a given silica value to temperature at which that composition may be expected to crystallize apatite. This model could be used for silica compositions between 45 and $75 \%$, and 0 and $10 \%$ water, and for the range of pressures expected in the crust (up to $25 \mathrm{kbar}$; Green and Adam 2002). Iron content or oxidation state of the liquid play insignificant effects on phosphate saturation (Tollari et al.2006). According 

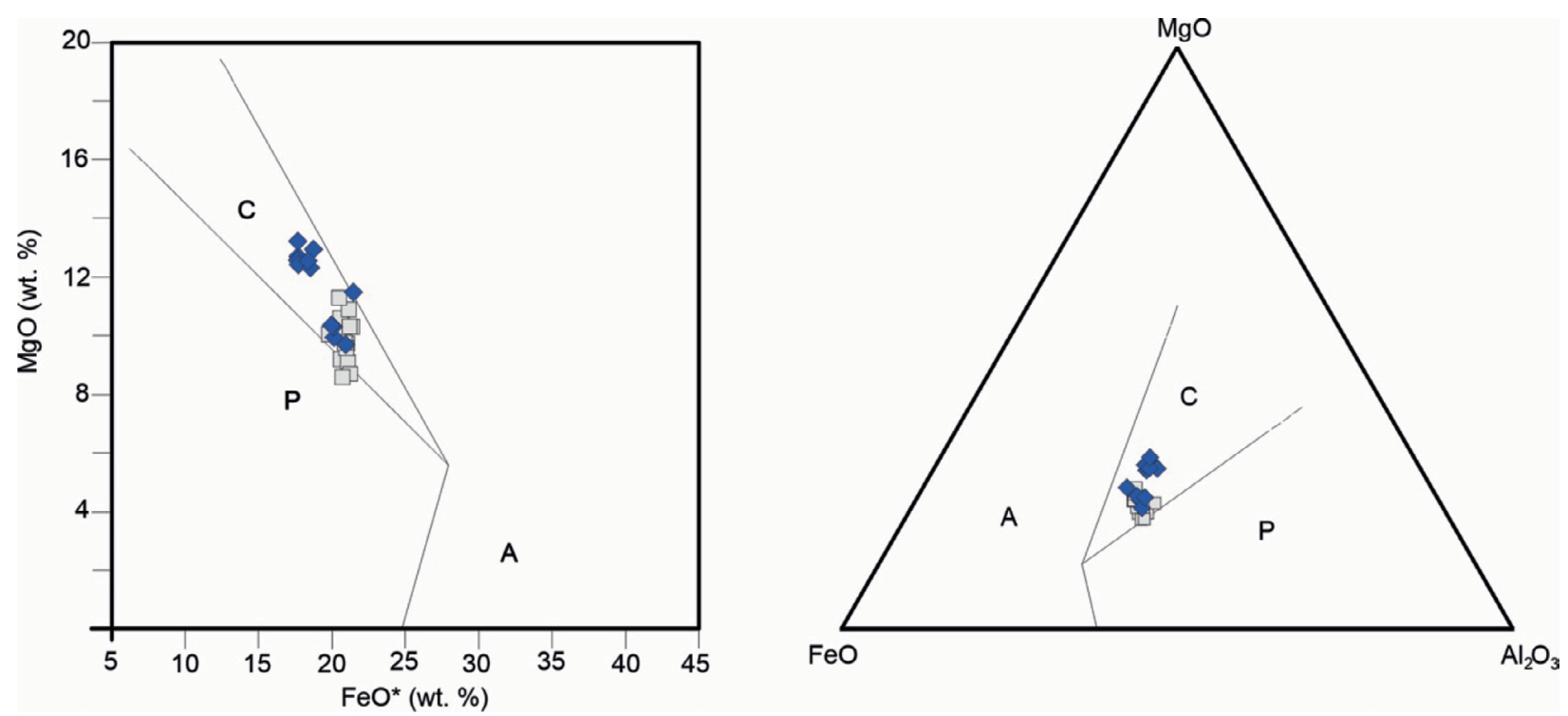

C: calc-alkaline; P: peraluminous; A: alkaline.

Figure 8. Composition of biotite from the studied granites expressed in the discrimination diagrams of Abdel-Rahman (1994). (A) MgO vs. $\mathrm{FeO}$ and (B) $\mathrm{MgO} \times \mathrm{FeO}_{x} \mathrm{Al}_{2} \mathrm{O}_{3}$. Symbols as in Fig. 3.

to Harrison and Watson (1984), the following expression can be used to estimate the minimum liquidus temperature: $\mathrm{T}\left({ }^{\circ} \mathrm{C}\right)=\left\{\left[8,400+26,400\left(\mathrm{SiO}_{2}-0.5\right)\right] /\left[\ln \left(42 / \mathrm{P}_{2} \mathrm{O}_{5}\right)+3.1\right.\right.$ $\left.\left.+12.4\left(\mathrm{SiO}_{2}-0.5\right)\right]-273.15\right\}$. Using this approach, the granites of the Major Isidoro pluton have near-liquidus temperatures that vary between 863 and $1,000^{\circ} \mathrm{C}\left(\right.$ average $\left.=936^{\circ} \mathrm{C}\right)$ while granites from the Monteirópolis pluton present a wider temperature range, from 814 to $1,023^{\circ} \mathrm{C}$ (Tab. 6). Apatite is an early phase relative to zircon in the crystallization sequence from both plutons, and apatite exhibits significantly higher saturation temperatures (e.g., Anderson et al. 2008, Naranjo and Vlach 2018) with an acceptable near-liquidus to solidus temperature span of $\sim 940$ to $\sim 700^{\circ} \mathrm{C}$.

\section{Amphibole barometry}

Several empirical and experimental igneous barometers based on Al-in-hornblende were proposed by Hammarstron and Zen (1986), Hollister et al. (1987), Schmidt (1992), Anderson and Smith 1995, Anderson 1996, Holland and Blundy (1994), and Mutch et al. (2016) to granitic rocks that have the mineral assemblage plagioclase $+\mathrm{K}$-feldspar + quartz + hornblende + biotite + titanite + magnetite \pm ilmenite + melt + fluid phase in equilibrium. The reported revised expression for the Al-in-hornblende barometer of Anderson and Smith (1995) incorporating the effect of temperature from the hornblende-plagioclase thermometer of Holland and Blundy (1994) and using the experimental data from Johnson and Rutherford (1989) and Schmidt (1992) was considered as reliable for our emplacement pressures. The resulting equation is Equation 2:

$$
\begin{aligned}
& \mathrm{P}( \pm 0.6 \mathrm{kbar})=4.76 \mathrm{Al}-3.01-\left\{\left[\mathrm{T}\left({ }^{\circ} \mathrm{C}\right)-675\right] / 85\right\} \mathrm{x} \\
& \left\{0.530 \mathrm{Al}+0.005294\left[\mathrm{~T}\left({ }^{\circ} \mathrm{C}\right)-675\right]\right\}
\end{aligned}
$$

Accordingly, when applied to the epidote-bearing Major Isidoro and Monteirópolis plutons, pressures of 5.27-7.71 and
4.3-5.34 kbar, respectively, are obtained. These results of the Al-inhornblende barometer of Schmidt (1992) and Anderson and Smith (1995) are portrayed in Table 2. These values are generally within the uncertainty of $\pm 0.6 \mathrm{kbar}$. These pressures of crystallization are within the lower limit of stability of magmatic epidote ( 0.3 to 0.7GPa; Zen and Hammarstrom 1984, Schmidt and Poli 2004).

\section{Oxygen fugacity}

Oxygen fugacity by far exerts the strongest control on mafic silicate mineral chemistry (Anderson and Smith 1995, Anderson 1996). This parameter is difficult to estimate in silicic rocks, especially those that contain only one Fe-Ti oxide mineral (Wones 1989). The presence of titanite + magnetite + quartz assemblage has long been known as suggestive of relatively high $\mathrm{fO}_{2}$ in siliceous magmas (e.g., Wones 1989, Enami et al. 1993, and references therein). According to Wones (1989), when this assemblage occurs along with clinopyroxene or amphibole with intermediate or higher $\mathrm{Mg} \#(\mathrm{Mg} /(\mathrm{Mg}+\mathrm{Fe}))$ ratios, relatively high $\mathrm{fO}_{2}$ is implied.

Anderson and Smith (1995) suggested that amphibole Fe\# ratios for barometry studies should be in the range of 0.40 0.65 (Mg-rich amphiboles). The amphibole from both Major Isidoro and Monteirópolis studied granites present Fe\# ratios that vary from $0.577-0.639$ and $0.552-0.575$, respectively, which are consistent with moderate to high oxygen fugacity (Fig. 9). This exceptional condition remains to be validated ( $c f$. Putirka 2016). Fe-rich amphibole bearing plutons that crystallized under low oxygen fugacity are not suitable for applying hornblende barometry, even though the full mineral assemblage is present and yields high pressures (Anderson and Smith 1995). Fe-rich amphiboles should not have $\mathrm{Fe}^{3+} /\left(\mathrm{Fe}^{3+}+\mathrm{Fe}^{2+}\right)$ ratios lower than 0.20 to apply hornblende barometry (Schmidt 1992, Anderson and Smith 1995). However, exceptions occur when biotite crystallize simultaneously with amphibole and partially incorporate Mg (e.g., Papoutsa and Pe-Piper 2014, Campos et al. 
2016). For the studied granites, hornblende $\mathrm{Fe}^{3+} /\left(\mathrm{Fe}^{3+}+\mathrm{Fe}^{2+}\right)$ ratios are within the limiting range except for one core and one rim of the analyzed amphibole from the Monteirópolis granite.

The whole-rock $\mathrm{Fe} \#[\mathrm{Fe} /(\mathrm{Fe}+\mathrm{Mg})]$ ratio varies independently of $\mathrm{Fe} \#$ ratio of amphibole and biotite, and with the increase of $\mathrm{fO}_{2}$, the $\mathrm{Fe} \#$ ratio of these minerals notably decrease (e.g., Anderson and Smith 1995, Anderson et al. 2008). Anderson et al. (2008) proposed an approximate $\mathrm{fO}_{2}$ relative to the quartz-fayalite-magnetite buffer $\left(\Delta_{\mathrm{OFM}}\right)$ depending upon $\mathrm{Fe} \#[\mathrm{Fe} /(\mathrm{Fe}+\mathrm{Mg})]$ in biotite. For biotite from the Major Isidoro granite, Fe\# in biotite ranges from 0.50 to 0.58 , and for biotite from the Monteirópolis from 0.43 to 0.55 , corresponding to $\Delta_{\mathrm{QFM}}=+1$ to $<+2.5$. This result is similar to those for granites in the northern Borborema Province obtained by Campos et al. (2016), who also observed that biotite with high $\mathrm{fO}_{2}\left(\Delta_{\mathrm{QFM}}=+0.8\right.$ to +2.0$)$ crystallizes together with amphibole at similar high oxidizing conditions.

The presence of magmatic epidote requires $\mathrm{fO}_{2}>$ quartz-fayalite-magnetite (QFM) (Liou 1973) and further reinforces

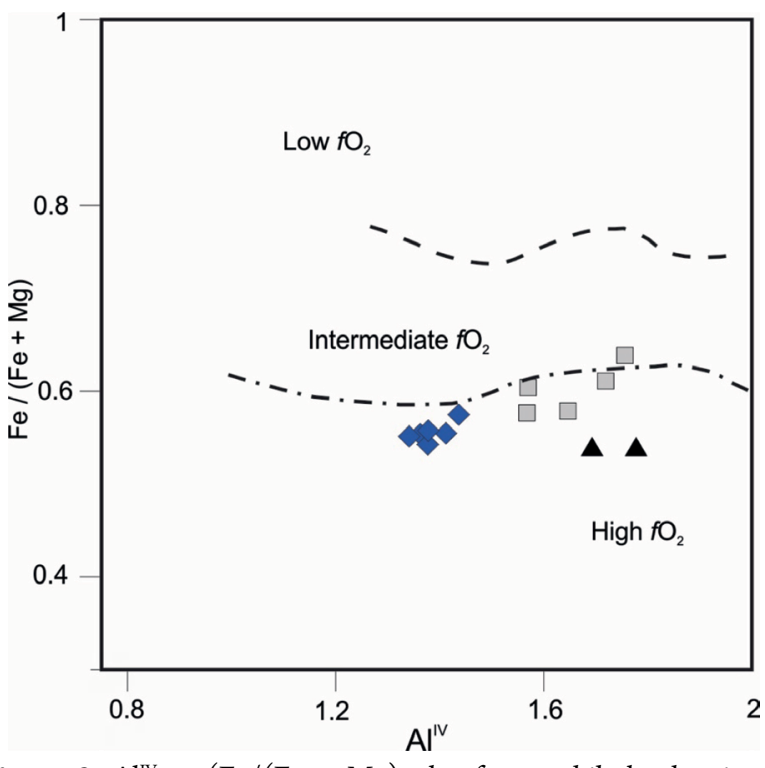

Figure 9. $\mathrm{Al}^{\mathrm{IV}}$ vs. $(\mathrm{Fe} /(\mathrm{Fe}+\mathrm{Mg})$ plot for amphibole showing qualitative $\mathrm{fO}_{2}$ conditions following Anderson and Smith (1995). Symbols as in Figure 3.

Table 6. Temperature estimates from apatite $\left(\mathrm{T}_{\mathrm{Ap}}\right)$ and zircon $\left(\mathrm{T}_{\mathrm{Zr}}\right)$ saturation thermometry. Major elements in wt.\%, and $\mathrm{Zr}$ in ppm.

\begin{tabular}{|c|c|c|c|c|c|c|c|c|c|c|c|c|}
\hline \multicolumn{13}{|c|}{ Major Isidoro pluton } \\
\hline Sample & UTM_E & UTM_N & $\mathrm{SiO}_{2}$ & $\mathrm{Al}_{2} \mathrm{O}_{3}$ & $\mathrm{CaO}$ & $\mathrm{Na}_{2} \mathrm{O}$ & $\mathbf{K}_{2} \mathbf{O}$ & $\mathbf{P}_{2} \mathbf{O}_{5}$ & $\mathbf{Z r}$ & $\mathbf{T}_{\mathrm{Zr}}\left({ }^{\circ} \mathbf{c}\right)$ & $\mathbf{T}_{\mathrm{Ap}}\left({ }^{\circ} \mathbf{C}\right)$ & $\mathbf{M}$ \\
\hline TR-13 & 715942 & 8943060 & 70.05 & 14.08 & 2.14 & 4.09 & 4.20 & 0.24 & 262 & 817 & 1000 & 1.57 \\
\hline TR-18 & 711590 & 8944776 & 68.87 & 14.43 & 2.69 & 3.51 & 3.58 & 0.26 & 408 & 868 & 998 & 1.47 \\
\hline TR-20 & 710825 & 8945732 & 67.08 & 14.41 & 2.88 & 4.01 & 2.12 & 0.09 & 95 & 740 & 863 & 1.43 \\
\hline TR-22 & 707957 & 8945926 & 59.13 & 16.90 & 4.58 & 5.24 & 2.07 & 0.49 & 364 & 824 & 972 & 1.86 \\
\hline TR 24 & 712856 & 8940776 & 69.23 & 15.39 & 1.42 & 3.36 & 5.10 & 0.11 & 317 & 857 & 906 & 1.30 \\
\hline TR-25 & 710959 & 8943026 & 68.68 & 14.91 & 2.41 & 3.55 & 4.95 & 0.17 & 305 & 832 & 947 & 1.55 \\
\hline TR-26 & 709992 & 8942174 & 67.86 & 15.34 & 3.25 & 4.62 & 1.59 & 0.14 & 120 & 756 & 917 & 1.47 \\
\hline TR-37 & 711823 & 8945606 & 66.58 & 15.62 & 2.36 & 3.66 & 4.60 & 0.17 & 281 & 831 & 927 & 1.48 \\
\hline TR-38 & 711168 & 8946056 & 65.61 & 15.40 & 2.84 & 4.10 & 2.20 & 0.22 & 273 & 836 & 944 & 1.38 \\
\hline TR-39 & 711239 & 8946774 & 65.09 & 16.10 & 3.05 & 4.90 & 2.00 & 0.25 & 260 & 822 & 954 & 1.50 \\
\hline \multicolumn{13}{|c|}{ Monteirópolis pluton } \\
\hline TR-01 & 706621 & 8945278 & 59.72 & 17.50 & 3.70 & 5.78 & 3.04 & 0.40 & 455 & 835 & 951 & 1.92 \\
\hline TR-43 & 698482 & 8946416 & 67.50 & 13.81 & 2.09 & 3.14 & 5.44 & 0.18 & 221 & 796 & 940 & 1.61 \\
\hline TR-45 & 699861 & 8944178 & 69.44 & 14.41 & 1.55 & 3.39 & 5.39 & 0.11 & 267 & 825 & 907 & 1.46 \\
\hline TR-46 & 700317 & 8943470 & 71.86 & 14.45 & 1.31 & 4.04 & 4.27 & 0.05 & 158 & 787 & 857 & 1.37 \\
\hline TR-48 & 700026 & 8941284 & 62.72 & 15.18 & 2.52 & 3.07 & 6.79 & 0.52 & 515 & 856 & 1023 & 1.83 \\
\hline TR-49 & 700693 & 8940170 & 71.49 & 15.82 & 1.18 & 4.20 & 4.66 & 0.09 & 128 & 772 & 908 & 1.32 \\
\hline TR-50 & 701528 & 8942522 & 73.43 & 14.56 & 0.74 & 3.87 & 5.05 & 0.03 & 112 & 763 & 828 & 1.31 \\
\hline TR-51 & 702705 & 8942624 & 71.27 & 15.19 & 1.06 & 4.42 & 4.47 & 0.13 & 121 & 764 & 944 & 1.37 \\
\hline TR-52 & 703283 & 8942420 & 64.53 & 17.14 & 2.43 & 5.12 & 3.77 & 0.45 & 388 & 848 & 1023 & 1.62 \\
\hline TR-73 & 706530 & 8944572 & 71.04 & 14.78 & 1.53 & 3.66 & 5.68 & 0.18 & 323 & 840 & 977 & 1.51 \\
\hline TR-74 & 706295 & 8944032 & 66.47 & 15.74 & 1.89 & 4.22 & 5.21 & 0.22 & 294 & 824 & 954 & 1.59 \\
\hline TR-76 & 705835 & 8941352 & 68.78 & 16.19 & 2.08 & 4.54 & 4.84 & 0.18 & 280 & 821 & 954 & 1.58 \\
\hline TR-77 & 704878 & 8941768 & 70.77 & 13.92 & 1.38 & 4.19 & 4.43 & 0.17 & 220 & 806 & 965 & 1.50 \\
\hline TR-78 & 703861 & 8942218 & 70.68 & 14.94 & 1.21 & 4.73 & 4.42 & 0.15 & 164 & 781 & 953 & 1.49 \\
\hline TR-79 & 702060 & 8943060 & 70.23 & 15.31 & 0.20 & 5.73 & 3.22 & 0.04 & 159 & 793 & 814 & 1.29 \\
\hline TR-80 & 701490 & 8943952 & 71.27 & 15.27 & 1.56 & 3.71 & 4.53 & 0.08 & 210 & 814 & 895 & 1.34 \\
\hline TR-85 & 702400 & 8945968 & 67.43 & 15.10 & 2.19 & 4.11 & 3.92 & 0.24 & 176 & 782 & 974 & 1.53 \\
\hline TR-88 & 703695 & 8947724 & 66.19 & 19.56 & 1.55 & 4.27 & 3.58 & 0.11 & 146 & 797 & 872 & 1.13 \\
\hline TR-89 & 705173 & 8946150 & 63.73 & 14.51 & 2.65 & 4.84 & 4.92 & 0.34 & 295 & 791 & 976 & 2.02 \\
\hline
\end{tabular}

Major elements in wt.\%, and $\mathrm{Zr}$ in ppm. $\mathrm{T}_{\mathrm{Zr}}\left({ }^{\circ} \mathrm{C}\right)=\{(12,900) /[\ln (497,644 / \mathrm{Zr})+3.8+0.85(\mathrm{M}-1)]-273.15\}$ where $\mathrm{M}=100(\mathrm{Na}+\mathrm{K}+2 \mathrm{Ca}) / \mathrm{Al} . \mathrm{Si}$. (Watson and Harrison 1983); $\mathrm{TAp}\left({ }^{\circ} \mathrm{C}\right)=\left\{\left[8,400+26,400\left(\mathrm{SiO}_{2}-0.5\right)\right] /\left[\ln \left(42 / \mathrm{P}_{2} \mathrm{O}_{5}\right)+3.1+12.4\left(\mathrm{SiO}_{2}-0.5\right)\right]-273.15\right\}$ (Harrison and Watson 1984). 
high oxygen fugacity. The pistacite content of epidote of the studied granites ( 27 to $31 \mathrm{~mol} . \%$ ) suggests that $\mathrm{fO}_{2}$ was higher than QFM and buffered between NNO (nickel-nickel oxide) and HM oxygen buffers (e.g., Sial et al. 2008).

\section{Granitic magma transport estimates}

Epidote from the Major Isidoro and Monteirópolis granites is partially included in later minerals such as biotite, plagioclase, and hornblende and shows resorbed rims, especially in contact with quartz and K-feldspar (Fig. 10). Epidote dissolution in residual melts is explained by non-equilibrium conditions when relatively high-pressure epidote is brought up from deeper levels in the magma and mineral aggregates shield and prevent re-equilibration of the epidote inclusions with residual melt (e.g., Brandon et al. 1996). The textural relationships of epidote with other minerals in rocks yield compelling information on upward magma transport (e.g., Brandon et al. 1996, Sial et al. 1999, 2008, Long et al. 2005, Brasilino et al. 2011). Sial et al. (2008), based on dissolution kinetic experiments on epidote from Brandon et al. (1996) and estimating the size of
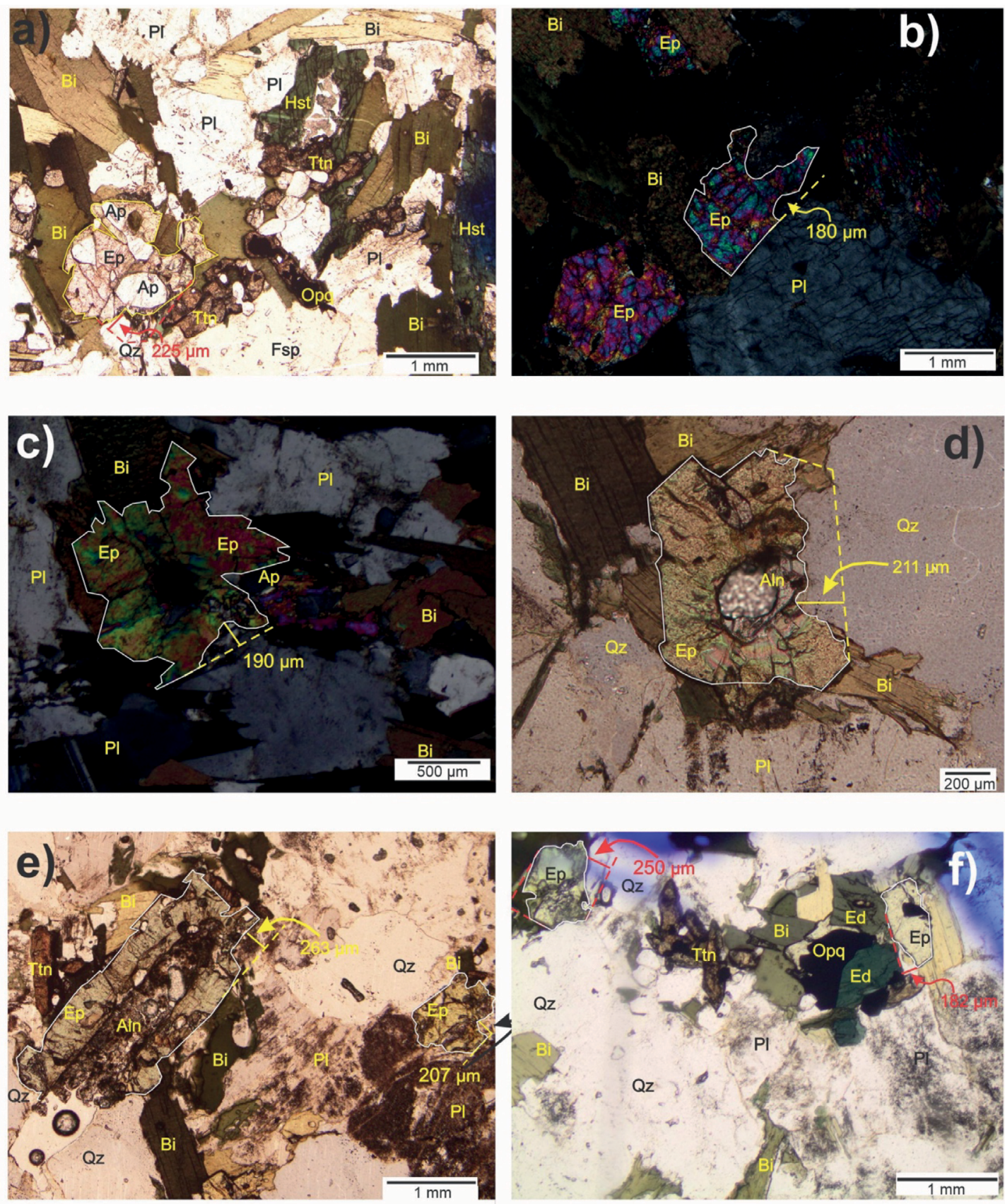

Figure 10. Textural aspects showed by magmatic epidote from the Major Isidoro (A, B, C) and Monteirópolis (D, E, F) granites. (A) Subhedral epidote armored by biotite; included occur euhedral apatite crystals; (B) subhedral epidote grains armored by biotite, and feldspar with minor corroded embayment; (C) euhedral magmatic epidote with allanite core included in biotite; (D) euhedral epidote partially dissolved by the host granitic magma; (E) euhedral magmatic epidote with allanite core embayed or totally dissolved where in contact with the quartz-feldspathic matrix; (F) euhedral epidote armored from total dissolution by biotite, and edenite from reaction with host magma. Parallel polarizers except for photo $\mathrm{b}$ and $\mathrm{c}$. dashed lines are an attempt to reconstruct original shape of epidote crystals, indicating how much some of them have been dissolved by host magma. Mineral abbreviations after Whitney and Evans (2010) are: All: allanite; Bt: biotite; Ep: epidote; Pl: plagioclase; Ttn: titanite; Ed: edenite; Hst: hastingsite; Ap: apatite; Qz: quartz; Opc: opaque mineral; Fsp: feldspar. 
partially corroded subhedral epidote crystals, proposed a way to estimate magma transport rates as follows:

- Selection of mEp based on their mol.\% Ps, and highly corroded subhedral grains which are partially shielded by plagioclase, biotite, or K-feldspar (Fig. 10);

- Inferring the original shapes of corroded grains and measure the maximum dissolution zone width;

- Estimating the duration of corrosion by using the minimum apparent diffusion coefficient of $5 \times 10^{-17} \mathrm{~m}^{2} / \mathrm{s}$ for $\mathrm{Si}, \mathrm{Al}, \mathrm{Ca}$, and $\mathrm{Fe}$ between tonalitic magma and epidote at $750^{\circ} \mathrm{C}$ (Brandon et al. 1996), as follows:

$$
\mathrm{d}_{\mathrm{z}}=\sqrt{ }\left[\mathrm{D}_{\text {app }} \mathrm{xt}\right]
$$

Where:

$\mathrm{d}_{\mathrm{z}}=$ width of dissolution zone $(\mathrm{m})$;

$\mathrm{D}_{\text {app }}=$ apparent diffusion coefficient $\left(5 \times 10^{-17} \mathrm{~m}^{2} / \mathrm{s}\right)$; and

$\mathrm{t}=$ time for partial dissolution of epidote $(\mathrm{s})$

Accordingly, $\mathrm{t}=\mathrm{d}_{\mathrm{z}}{ }^{2} /\left(5 \times 10^{-17} \mathrm{~m}^{2} / \mathrm{s}\right)$.

- Depth of host magma emplacement is inferred from Al-in hornblende barometry;

- The rate of magma transport is the ratio of the route length (the difference between the emplacement depth and the source depth) to the average time of corrosion of epidote exposed to the host melt. For a tonalite melting, at water-saturated conditions and $\mathrm{fO}_{2}$ buffered by NNO, plagioclase and epidote may coexist from a depth $\sim 10 \mathrm{kbar}$ (Schmidt and Thompson 1996, Fig. 2). We infer the route length as the difference between $10 \mathrm{kbar}$ and the emplacement depth. Accordingly,

$$
\mathrm{T}_{\mathrm{r}}=\mathrm{L}_{\mathrm{r}} / \mathrm{t}
$$

Where:

$\mathrm{T}_{\mathrm{r}}=$ transport rate $(\mathrm{m} /$ year $)$;

$\mathrm{L}_{\mathrm{r}}=\left(10-\mathrm{P}_{\mathrm{e}}\right) \cdot 10^{4} / 3(\mathrm{~m})$;

$\mathrm{L}_{\mathrm{r}}=$ length route $(\mathrm{m})$;

$\mathrm{P}_{\mathrm{e}}=$ pressure of emplacement $(\mathrm{kbar})$;

$\mathrm{t}=$ time of partial dissolution of epidote (year).

Based on this approach estimated by Sial et al. (2008), we obtain the time for the dissolved width of magmatic epidote and the maximum upward ascension rates of the magmas that formed the Major Isidoro and Monteirópolis granites. This method gives only maximum speed because the calculations assume implicitly that epidote starts reacting with the host magma as pressure drops below $10 \mathrm{kbar}$ assuming, therefore, the minimum possible time for corrosion of epidote crystals. For the Major Isidoro granites, the measured width of dissolution of 0.157 to $0.225 \mathrm{~mm}$ occurred during 15.6 to 32 years of partial corrosion, which correspond to ascent rates of 365 to 750 m.year $^{-1}$, while for the Monteirópolis granites, the measured dissolution zones of 0.207 to $0.263 \mathrm{~mm}$ occurred during $27-49$ years of partial corrosion, which correspond to ascent rates of 395 to 635 m.year $^{-1}$.

Based on this approach, some limiting assumptions are found as pointed out by Sial et al. (2008):
- The error brackets on the Al-in-hornblende barometry, typically of $\pm 0.6 \mathrm{kbar}$;

- Use of appropriate diffusion coefficients for $\mathrm{Ca}, \mathrm{Al}, \mathrm{Si}$, and Fe for each magma composition;

- Bias in measuring dissolution zone widths of mEp grains (only subhedral grains were used in this study and anhedral grains were ignored);

- Decreasing rate of epidote dissolution, leading to an underestimate of digestion time.

Despite these uncertainties, the method provides a good general indication of rapid magma ascent. This is primarily due to the very short calculated dissolution times, which is $<50 \mathrm{yr}$. When compared with ascent timescale from the literature, our rapid rate of upward magma movement suggests diking/conduit flow $\left(10^{-1}-10^{2}\right.$ years $)$, and not diapirism $\left(10^{6}-10^{9}\right.$ years $)$, as the ascent mechanism ( $c f$. Clemens and Mawer 1992, Petford et al. 1993, 2000). Our calculated dissolution times can be increased by four orders of magnitude and still be short.

\section{Magmatic epidote-bearing granites elsewhere}

The occurrence of magmatic Epidote $(\mathrm{mEp})$ and amphibole are key features to characterize the $\mathrm{P}-\mathrm{T}-\mathrm{fO}_{2}$ conditions and many textural features have been considered to identify epidote as magmatic (Zen and Hammarstrom 1984, Schmidt and Poli 2004, Sial et al. 1999, 2008), such as:

- Strong zonation with allanite rich cores;

- Inclusion of mEp into biotite, amphibole, and plagioclase;

- Poikilitic texture;

- Lack of biotite alteration to chlorite;

- Lack of plagioclase alteration.

Additionally, compositional criteria (e.g., Tulloch 1979) based on pistacite contents have been used to differentiate its magmatic of subsolidus origin. Magmatic epidote $(\mathrm{mEp})$ in granitic rocks is known since its early description by Keyes (1983a, 1983b), however, just from the 1980s has its occurrence been recorded worldwide (e.g. North America: Hammarstron and Zen 1986; Europe: Terentiev et al. 2016; Africa: Tchameni et al. 2016; India: Rogers 1988, Basaket al. 2019; NewZealand: Tulloch 1979; South America: Sial 1986, Sial and Ferreira 1990,2015, Sial et al. 1997, 1998, 2008).

\section{Epidote-bearing granite transport and formation in the Borborema Province}

In the Borborema Province, northeastern Brazil, epidote-bearing granites occur commonly throughout Cryogenian-Ediacaran plutons (see Sial and Ferreira 2015). Interestingly, plutons of similar chemical composition, crystallized at similar pressure, may or may not contain magmatic epidote (e.g., Sial et al. 2008). This mineral is highly vulnerable to changing $\mathrm{P}-\mathrm{T}$ conditions by dissolving during magma ascent; however, the presence of $\mathrm{mEp}$ in granitic rocks allows constraining the depth of melting and crystallization, and ascension rates through the continental crust. In this province, magmatic epidote is more abundant and better preserved in older $(0.64-0.62 \mathrm{Ga})$ plutons, however, it occurs in fewer younger (ca. $0.57 \mathrm{Ga}$ ) plutons ( $c f$. Ferreira et al. 
2004). Ferreira et al. (2011) tied the magmatic epidote survival, which is possible only when upward magma transport from the deep crust is rapid, in the older plutons of the Transversal Domain of the Borborema Province, to hot continental crust conditions during the peak of metamorphism, in the main stage of the Brasiliano orogenic cycle. The Major Isidoro and Monteirópolis plutons are two of these early plutons $(\sim 0.63 \mathrm{Ga}$; Silva et al. 2015, 2016) plausibly produced by partial melting of a reworked Tonian lower-continental crust and by Tonian mantle-derived rocks, respectively, emplaced during the Brasiliano orogenesis along the JHSZ, which must have acted to facilitate the fast upward migration that contributed to prevent $\mathrm{mEp}$ dissolution. This is consistent with buoyancy-ascent of magmas in narrow channels, for instance; via an interconnected network of shear zones, as suggested by Collins and Sawyer (1996) and Petford et al. (2000). Narrow conduits (e.g., dikes) ascent has been shown a viable mechanism for the transport of large volumes of calc-alkalic granite melt through the continental crust during a short timescale (Petford et al. 1993).

De Saint Blanquat et al. (2011) found a pluton volume of first-order importance for the duration of pluton construction and observed that the larger a pluton, the longer its construction time. Roughly considering the volume of the studied plutons between 100 and $200 \mathrm{~km}^{3}$ would give construction time span duration of less than $10^{5}$ years (Petford et al. 2000, De Saint Blanquat et al. 2011, and references therein), which is fairly higher than our fast ascent rates requiring more data (e.g., geophysical to better know the 3-d geometry and accurate volume of the studied pluton) to better understand and discuss. However, this contrast could be partly solved if we consider the growing magmatic pluton source-controlled by magma batches. As showed by Solano et al. (2012), lateral migration of evolved magma through high-porosity melt layers can collect the evolved granitic magma generated from a deep crustal hot zone (DCHZ) of larger areal extent into a localized ascent zone comprising dikes, faults, or shear zones. The segregation of granitic magma from its source is strongly dependent on its physical properties, of which viscosity is the most important one as a function of composition, temperature, and water content (Barker 1998, Giordano et al. 2008, and references therein). This property is a critical quantity governing transport ascent in volcanic and magmatic processes. The viscosities of the Major Isidoro and Monteirópolis magmas over their range of composition, water contents, and temperature (59.1-73.4 wt $\% \mathrm{SiO}_{2}, 0.36-1.5 \mathrm{wt} \% \mathrm{H}_{2} \mathrm{O}$, and $800-1,000^{\circ} \mathrm{C}$, respectively; Tab. 6) were calculated using the model of Giordano et al. (2008), which agreed with subsequent experiments on felsic-mafic magma mixing reality (e.g., Laumonier et al. 2014), providing values of $10^{4.1}-10^{8.4}$, and $10^{4.2}-10^{8.5} \mathrm{~Pa}$ $\mathrm{s}$, respectively (lower than $10^{6} \mathrm{~Pa}$ s to near-liquidus temperatures of $\sim 1,000^{\circ} \mathrm{C}$ ). Our viscosities values are within those found in the literature for granitic and volcanic magmas that are generally in the range of $10^{3}-10^{8} \mathrm{~Pa}$ s (Scaillet et al. 1998, Clemens and Petford 1999, Laumonier et al. 2014), which

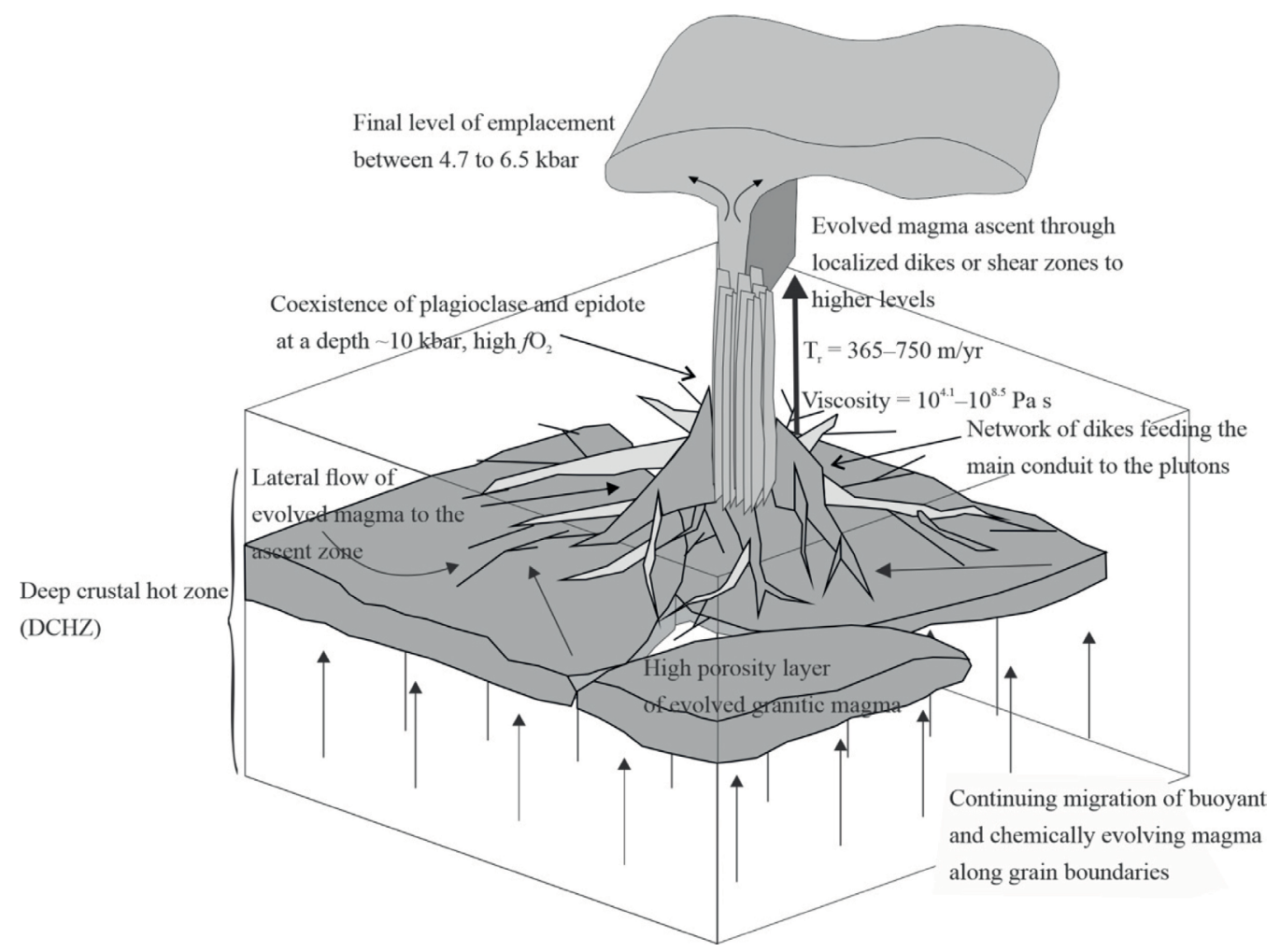

Figure 11. Schematic block diagram synthesizing the discussion in the text, from the place of partial melting in a deep crustal hot zone (DCHZ) to the level of emplacement of the Major Isidoro and Monteirópolis plutons (modified from Solano et al. 2012). The drawing is not to scale. After partial melting, the evolved magma may flow laterally and be drained via a network of dikes feeding a main conduit (e.g., dikes, faults, or shear zones) to the plutons (e.g., Brown and Solar 1998, Vanderhaeghe 1999, 2001, 2009, Hall and Kisters 2012). We consider watersaturated conditions and $\mathrm{fO}_{2}$ buffered by NNO, where plagioclase and epidote may coexist at a depth $\sim 10 \mathrm{kbar}$. From the ascent route of this depth to the level of emplacement, partial dissolution of epidote occurs during very short calculated dissolution times $(<50 \mathrm{yr})$. 
evidence very rapid magma ascent rates of up to $1 \mathrm{~m} . \mathrm{s}^{-1}$ (e.g., Castro and Dingwell 2009). In Figure 11, we propose a schematic block diagram synthesizing our finds from the place of partial melting in a DCHZ to the level of emplacement of the Major Isidoro and Monteirópolis plutons.

\section{CONCLUSIONS}

The Major Isidoro (627 Ma) and Monteirópolis (626 $\mathrm{Ma}$ ) granites crystallized under oxidizing conditions between the NNO-HM buffers, with initial crystallization temperatures greater than $940^{\circ} \mathrm{C}$. Pressures of 5.3-7.7 kbar (Major Isidoro) and 4.3-5.3 kbar (Monterirópolis) were estimated for the final level of emplacement. Epidote survival (partially dissolved) evidences rapid upward transportation rates of the Major Isidoro and Monteiropólis magmas (365-750 m/ year), which were probably favored by the JHSZ, through hot deep to upper continental crust during the onset of the Brasiliano orogeny. These rates are comparable to those from epidote-bearing granites of similar age in other domains of the Borborema Province.

\section{ACKNOWLEDGMENTS}

We thank Edward Sawyer for discussions on the geology of the Major Isidoro pluton. Nilson M. Botelho is thanked for mineral chemistry laboratory facilities. We are grateful to Bruna Maria Borba de Carvalho for the acquisition of mineral chemistry at the Electron Microprobe Laboratory in the Universidade de Brasília, Brazil, and Anderson de Abreu da Silva for the XRF chemical analyses at the Stable Isotope Laboratory (LABISE) in the Universidade Federal de Pernambuco, Brazil. Comments on drafts and revised versions of the manuscript by Maia de Lourdes, Anelise Bertoti, Julio Mendes, and Adejardo da Silva Filho, as members of Silva's thesis committee, are acknowledged. We are grateful to Claudio Ricomini, Chief Editor, for careful editorial handling, and especially to Olivier Vanderhaeghe, Silvio Vlach, and an anonymous reviewer for their positive, critical, and constructive comments, which greatly contribute to improve the paper. Obviously, any errors or omissions are solely the responsibility of the authors. VPF and ANS acknowledge the continuous financial support from the $\mathrm{CNPq}$ (process number 471034/2012-6) and FACEPE (process APQ-1738-1.07/12). This is the NEG-LABISE contribution n. 293.

\section{ARTICLE INFORMATION}

Manuscript ID: 20190110. Received on: 10/15/2019. Approved on: 04/30/2020.

T.S. idealized and wrote the first draft of the manuscript, and prepared the model and figures; M.L. partly wrote the manuscript and helped with the figures; V.F. and A.S. provided valuable advisorship regarding petrology and mineral chemistry, and provided inputs and modifications for the text and concepts. The entire manuscript was revised, commented, and corrected by all co-authors.

Competing interests: The authors declare no competing interests.

\section{REFERENCES}

Abdel-Rahman A.F.M. 1994. Nature of Biotites from Alkaline, Calcalkaline, and Peraluminous Magmas. Journal of Petrology, 35(2):525-541. https://doi.org/10.1093/petrology/35.2.525

Amorim J.V.A., Guimarães I.P., Farias D.J.S., Lima J.V., Santos L., Ribeiro V.B., Brainer C. 2019. Late-Neoproterozoic ferroan granitoids of the Transversal subprovince, Borborema Province, NE Brazil: petrogenesis and geodynamic implications. International Geology Review, 61(14):1745-1767. https://doi.org/10.1080/00206814.2018.1544936

Anderson J.L. 1996. Status of thermobarometry in granitic batholiths. Transactions of the Royal Society of Edinburgh: Earth Sciences, 87(1-2):125-138. https://doi.org/10.1017/S0263593300006544

Anderson J.L., Barth A.P., Wooden J.L., Mazdab F. 2008. Thermometry and Thermobarometers in Granitic Systems. Reviews in Mineralogy and Geochemistry, 69(1):121-142. https://doi.org/10.2138/rmg.2008.69.4

Anderson J.L., Smith D.R. 1995. The effects of temperature and $\mathrm{fO}_{2}$ on the Al-in-hornblende barometer. American Mineralogist, 80(5-6):549-559. https://doi.org/10.2138/am-1995-5-614

Bachmann O., Dungan M. 2002. Temperature-induced Al-zoning in hornblendes of the Fish Canyon magma, Colorado. American Mineralogist, 87(8-9):1062-1076. https://doi.org/10.2138/am-2002-8-903

Barker D.R. 1998. Granitic melt viscosity and dike formation. Journal of Structural Geology, 20(9-10):1395-1404. https://doi.org/10.1016/ S0191-8141(98)00057-1

Basak A., Goswami B., Singha A., Das S., Bhattacharyya C. 2019. Magmatic epidote in the Grenvillian granitoids of North Purulia Shear Zone, Chhotanagpur Gneissic Complex, India and its significance. Current Science, 117(2):298-303. https://doi.org/10.18520/cs/v117/i2/298-303

Blundy J.D., Holland T.J.B. 1990. Calcic amphibole equilibria and a new amphibole-plagioclase geothermometer. Contributions to Mineralogy and Petrology, 104:208-224. https://doi.org/10.1007/BF00306444
Boehnke P., Watson E.B., Trail D., Harrison T.M., Schmitt A. 2013. Zircon saturation re-revisited. Chemical Geology, 351:324-334. https://doi. org/10.1016/j.chemgeo.2013.05.028

Brandon A.D., Creaser R.A., Chacko T. 1996. Constraints on Rates of Granitic Magma Transport from Epidote Dissolution Kinetics. Science, 271(5257):1845-1848. https://doi.org/10.1126/science.271.5257.1845

Brasilino R.G., Sial A.N., Ferreira V.P., Pimentel M.M. 2011. Bulk rock and mineral chemistries and ascent rates of high-K calc-alkalic epidote-bearing magmas, Northeastern Brazil. Lithos, 127(3-4):441-454. https://doi. org/10.1016/j.lithos.2011.09.017

Brasilino R.G., Sial A.N., Lafon J.M. 1999. Magmatic epidote, hornblende barometric estimates, and emplacement of the Conceição das Creoulas pluton, Alto Pajeu Terrane, NE Brazil. Anais da Academia Brasileira de Ciências, 71(1):3-16.

Brito M.F.L., Silva Filho A.F., Guimarães I.P. 2009. Geologia isotópica do batólito shoshonítico-ultrapotássico Neoproterozóico Serra do Catú e implicações na evolução da interface dos Domínios Canindé e PernambucoAlagoas. Revista Brasileira de Geociências, 39(2):324-337. https://doi. org/10.25249/0375-7536.2009392324337

Brown M., Solar G.S. 1998. Granite ascent and emplacement during contractional deformation in convergent orogens. Journal of Structural Geology, 20(9-10):1365-1393. https://doi.org/10.1016/ S0191-8141(98)00074-1

Campos B.C.S., Vilalva F.C.J., Nascimento M.A.L., Galindo A.C. 2016 Crystallization conditions of porphyritic high-K calc-alkaline granitoids in the extreme northeastern Borborema Province, NE Brazil, and geodynamic implications. Journal of South American Earth Sciences, 70:224-236. https:// doi.org/10.1016/j.jsames.2016.05.010

Castro J.M., Dingwell D.B., 2009. Rapid ascent of rhyolitic magma at Chaitén volcano, Chile. Nature, 461:780-783. https://doi.org/10.1038/ nature 08458 
Clemens J.D., Mawer C.K. 1992. Granitic magma transport by fracture propagation. Tectonophysics, 204(3-4):339-360. https://doi. org/10.1016/0040-1951(92)90316-X

Clemens J.D., Petford N. 1999. Granitic melt viscosity and silicic magma dynamics in contrasting tectonic settings. Journal of the Geological Society, 156(6):1057-1060. https://doi.org/10.1144/gsigs.156.6.1057

Collins W.J., Sawyer E.W. 1996. Pervasive granitoid magma transfer through the lower-middle crust during non-coaxial compressional deformation. Journal of Metamorphic Geology, 14(5):565-579. https://doi. org/10.1046/j.1525-1314.1996.00442.x

Deer W.A., Howie R.A., Zussman J. 2013. An Introduction to the RockForming Minerals. $3^{\text {rd }}$ ed. London: Mineralogical Society, 498 p. https://doi. org/10.1180/DHZ

De Oliveira R.G. 2008. Arcabouço geofísico, isostasia e causas do magmatismo Cenozóico da Província Borborema e de sua margem continental (Nordeste do Brazil). $\mathrm{PhD}$ thesis, Federal University of Rio Grande do Norte, Natal, 411 p.

De Saint Blanquat M., Horsman E., Habert G., Morgan S., Vanderhaeghe O., Law R., Tikoff B. 2011. Multiscale magmatic cyclicity, duration of pluton construction, and the paradoxical relationship between tectonism and plutonism in continental arcs. Tectonophysics, 500(1-4):20-33. https://doi. org/10.1016/j.tecto.2009.12.009

Enami M., Suzuki K., Liou J.G., Bird D.K. 1993. Al-Fe ${ }^{3+}$ and F-OH substitutions in titanite and constraints on their $\mathrm{P}-\mathrm{T}$ dependence. European Journal of Mineralogy, 5(2):219-231. https://doi.org/10.1127/ ejm/5/2/0219

Evans B.W., Vance J.A. 1987. Epidote phenocrysts in dacitic dikes, Boulder County, Colorado. Contributions to Mineralogy and Petrology, 96:178-185. https://doi.org/10.1007/BF00375231

Ferreira V.P., Sial A.N., Jardim de Sá E.F. 1998. Geochemical and isotopic signatures of the Proterozoic granitoids in terranes of the Borborema Province, northeastern Brazil. Journal of South American Earth Sciences, 11(5):439-455. https://doi.org/10.1016/S0895-9811(98)00027-3

Ferreira V.P., Sial A.N., Pimentel M.M., Armstrong R., Guimarães I.P., Silva Filho A.F., Lima M.M.C., Silva T.R. 2015. Reworked old crust-derived shoshonitic magma: The Guarany pluton, Northeastern Brazil. Lithos, 232:150-161. https://doi.org/10.1016/j.lithos.2015.06.030

Ferreira V.P., Sial A.N., Pimentel M.M., Armstrong R., Spicuzza M.J., Guimarães I.P., Silva Filho A.F. 2011. Contrasting sources and P-T crystallization conditions of epidote-bearing granitic rocks, northeastern Brazil: O, Sr, and Nd Isotopes. Lithos, 121(1-4):189-201. https://doi. org/10.1016/j.lithos.2010.11.002

Ferreira V.P., Sial A.N., Pimentel M.M., Moura C.A.V. 2004. Acidic to intermediate magmatism and crustal evolution in the Transversal Zone, northeastern Brazil. In: Mantesso Neto V., Bartorelli A., Carneiro C.D.R., Brito Neves B.B. (Eds.). Geologia do Continente Sul-americano: a evolução da obra de Fernando Flávio Marques de Almeida. São Paulo: Editora USP, p. 189-201.

Ferreira V.P., Valley J.W., Sial A.N, Spicuzza M.J. 2003. Oxygen isotope compositions and magmatic epidote from two contrasting metaluminous granitoids, NE Brazil. Contributions to Mineralogy and Petrology, 145:205-216. https://doi.org/10.1007/s00410-003-0443-4

Giordano D., Russell J.K., Dingwell D.B. 2008. Viscosity of magmatic liquids: A model. Earth and Planetary Science Letters, 271(1-4):123-134. https://doi.org/10.1016/j.epsl.2008.03.038

Green T.H., Adam J. 2002. Pressure effect on Ti- or P-rich accessory mineral saturation in evolved granitic melts with differing $\mathrm{K}_{2} \mathrm{O} / \mathrm{Na}_{2} \mathrm{O}$ ratios. Lithos, 61(3-4):271-282. https://doi.org/10.1016/S0024-4937(02)00083-X

Green T.H., Watson E.B. 1982. Crystallization of Apatite in Natural Magmas Under High Pressure, Hydrous Conditions, with Particular Reference to 'Orogenic' Rock Series. Contributions to Mineralogy and Petrology, 79:96-105. https://doi.org/10.1007/BF00376966

Guimarães I.P., Silva Filho A.F., Almeida C.N., Van Schmus W.R., Araújo J.M.M., Melo S.C., Melo E.B. 2004. Brasiliano (Pan-African) granitic magmatism in the Pajeú-Paraíba belt, Northeast Brazil: an isotopic and geochronological approach. Precambrian Research, 135(1-2):23-53. https://doi.org/10.1016/j.precamres.2004.07.004
Hall D., Kisters A. 2012. The stabilization of self-organised leucogranite networks-Implications for melt segregation and far-field melt transfer in the continental crust. Earth and Planetary Science Letters, 355-356:1-12. http://doi.org/10.1016/j.epsl.2012.08.033

Hammarstron J.M., Zen E.-A. 1986. Aluminum in hornblende: An empirical igneous geobarometer. American Mineralogist, 71(11-12):1297-1313.

Harrison T.M., Watson E.B. 1984. The behavior of apatite during crustal anatexis: Equilibrium and kinetic considerations. Geochimicaet Cosmochimica Acta, 48(7):1467-1477. https://doi.org/10.1016/0016-7037(84)90403-4

Hawthorne F.C., Oberti R., Harlow G.E., Maresch W.V., Martin R.F., Schumacher J.C., Welch M.D. 2012. IMA Report: Nomenclature of the amphibole supergroup. American Mineralogist, 97(11-12):2031-2048. https://doi.org/10.2138/am.2012.4276

Holland T., Blundy J. 1994. Non-ideal interactions in calcic amphiboles and their bearing on amphibole-plagioclase thermometry. Contributions to Mineralogy and Petrology, 116:433-447. https://doi.org/10.1007/ BF00310910

Hollister L.S., Grissom G.C., Peters E.K., Stowell H.H., Sisson V.B. 1987. Confirmation of the empirical correlation of $\mathrm{Al}$ in hornblende with pressure of solidification of calc-alkaline plutons. American Mineralogist, 72(3-4):231-239.

Johnson M.C., Rutherford M.J. 1989. Experimental calibration of the aluminum-in-hornblende geobarometer with application to Long Valley caldera (California) volcanic rocks. Geology, 17(9):837-841. https://doi. org/10.1130/0091-7613(1989)017<0837:ECOTAI>2.3.CO;2

Johnston A.D., Wyllie P.J. 1988. Interaction of granitic and basic magmas: experimental observations on contamination processes at $10 \mathrm{kbar}$ with $\mathrm{H}_{2} \mathrm{O}$. Contributions to Mineralogy and Petrology, 98:352-362. https://doi. org/10.1007/BF00375185

Keyes C.R. 1983a. Epidote as a primary component of eruptive rocks. Geological Society of America Bulletin, 4(1):305-312. https://doi. org/10.1130/GSAB-4-305

Keyes C.R. 1983b. Some Maryland Granites and their Origin. Geological Society of America Bulletin, 4(1):299-304. https://doi.org/10.1130/ GSAB-4-299

Laumonier M., Scaillet B., Pichavant M., Champallier R., Andujar J., Arbaret L. 2014. On the conditions of magma mixing and its bearing on andesite production in the crust. Nature Communications, 5:5607. https://doi. org/10.1038/ncomms6607

Leake B.E., Woolley A.R., Arps C.E.S., Birch W.D., Gilbert M.C., Grice J.D., Hawthorne F.C., Kato A., Kisch H.J., Krivovichev V.G., Linthout K., Laird J., Mandarino J., Maresch W.V., Nickel E.H., Rock N.M.S., Schumacher J.C., Smith D.C., Stephenson N.C.N., Ungaretti L., Whittaker E.J.W., Youzhi G. 1997. Nomenclature of amphiboles: Report of the Subcommittee on Amphiboles of the International Mineralogical Association Commission on New Minerals and Mineral Names. European Journal of Mineralogy, 9(3):623-651.

Leake B.E., Woolley A.R., Birch W.D., Burke E.A.J., Ferraris G., Grice J.D., Hawthorne F.C., Kisch H.J., Krivovichev V.G., Schumacher J.C., Stephenson N.C.N., Whittaker E.J.W. 2003. Nomenclature of amphiboles: Additions and revisions to the International Mineralogical Association's 1997 recommendations. The Canadian Mineralogist, 41(6):1355-1362. https:// doi.org/10.2113/gscanmin.41.6.1355

Lima J.V., Guimarães I.P., Santos L., Amorim J.V.A., Farias D.J.S. 2017. Geochemical and isotopic characterization of the granitic magmatism along the Remígio - Pocinhos shear zone, Borborema Province, NE Brazil. Journal of South American Earth Sciences, 75:116-133. https://doi.org/10.1016/j. jsames.2017.02.004

Lima M.M.C. 2019. Petrologia e geoquímica de plútons do leste do batólito Águas Belas-Canindé, Domínio Pernambuco-Alagoas, Província Borborema. PhD thesis, Universidade Federal de Pernambuco, Recife, 206 p.

Lima M.M.C., Silva T.R., Ferreira V.P., Silva J.M.R. 2014. Metasedimentary rocks of the northern portion of the Macururé domain, Sergipano Belt, northeastern Brazil: Geochemical characterization of their protoliths and tectonic implications. Estudos Geológicos, 24(2):89-107. https://doi. org/10.18190/1980-8208/estudosgeologicos.v24n2p89-107 
Liou J.G. 1973. Synthesis and stability relations of epidote, $\mathrm{Ca}_{2} \mathrm{Al}_{2} \mathrm{FeSiO}_{12}(\mathrm{OH})$. Journal of Petrology, 14(3):381-413. https://doi. org/10.1093/petrology/14.3.381

Long E.L., Castellana C.H., Sial A.N. 2005. Age, Origin and Cooling History of the Coronel João Sá Pluton, Bahia, Brazil. Journal of Petrology, 46(2):255-273. https://doi.org/10.1093/petrology/egh070

Long E.L., Ketcham D.H., Fanning C.M., Sial A.N. 2019. The unregenerate São Rafael pluton, Borborema Province, Northeastern Brazil. Lithos, 332-333:192-206. https://doi.org/10.1016/j.lithos.2019.01.036

Mendes V., Brito M.F.L., Paiva I.P. 2009. Folha Arapiraca (SC.24-X-D, escala 1:250.000). Integration Geological (Geologic map). Recife: Brazilian Geological Survey (CPRM).

Miller C.F., McDowell S.M., Mapes R.W. 2003. Hot and cold granites? Implications of zircon saturation temperatures and preservation of inheritance. Geology, 31(6):529-532. https://doi. org/10.1130/0091-7613(2003)031<0529:HACGIO>2.0.CO;2

Molina J.F., Moreno J.A., Castro A., Rodríguez C., Fershtater G.B. 2015. Calcic amphibole thermobarometry in metamorphic and igneous rocks: New calibrations based on plagioclase/amphibole Al-Si partitioning and amphibole/liquid $\mathrm{Mg}$ partitioning. Lithos, 232:286-305. https://doi. org/10.1016/j.lithos.2015.06.027

Mutch E.J.F., Blundy J.D., Tattitch B.C., Cooper F.J., Brooker R.A. 2016. An experimental study of amphibole stability in low-pressure granitic magmas and a revised Al-in-hornblende. Contributions to Mineralogy and Petrology, 171:85. https://doi.org/10.1007/s00410-016-1298-9

Nachit H., Razafimahefa N., Stussi J.M., Carron J.P. 1985. Composition chimique des biotites et typologie magmatique des granitoïdes. Comptes rendus de l'Académie des sciences de la Terre et des planetes. Earth and Planetary Sciences, 11 (II):813-818.

Naranjo A.F.S., Vlach S.R.F. 2018. On the crystallization conditions of the Neoproterozoic, high-K calc-alkaline, Bragança Paulista-type magmatism, southern Brasília Orogen, SE Brazil. Brazilian Journal of Geology, 48(3):631-650. https://doi.org/10.1590/2317-4889201820180033

Neves S.P., Bruguier O., Vauchez A., Bosch D., Silva J.M.R., Mariano G. 2006. Timing of crust formation, deposition of supracrustal sequences, and Transamazonian and Brasiliano metamorphism in the East Pernambuco belt (Borborema Province, NE Brazil): Implications for western Gondwana assembly. Precambrian Research, 149(3-4):197-216. https://doi. org/10.1016/j.precamres.2006.06.005

Papoutsa A., Pe-Piper G. 2014. Geochemical variation of amphiboles in A-type granites as an indicator of complex magmatic systems: Wentworth pluton, Nova Scotia, Canada. Chemical Geology, 384:120-134. https://doi. org/10.1016/j.chemgeo.2014.07.001

Petford N., Cruden A.R., McCaffrey K.J.W., Vigneresse J.-L. 2000. Granite magma formation, transport and emplacement in the Earth's crust. Nature, 408:669-673. https://doi.org/10.1038/35047000

Petford N., Kerr R.C., Lister J.R. 1993. Dike transport of granitoid magmas. Geology, 21(9):845-848. https://doi. org/10.1130/0091-7613(1993)021<0845:DTOGM >2.3.CO;2

Putirka K. 2016. Amphibole thermometers and barometers for igneous systems and some implications for eruption mechanisms of felsic magmas at arc volcanoes. American Mineralogist, 101(4):841-858. https://doi. org/10.2138/am-2016-5506

Rogers J.J.W. 1988. The Arsikere Granite of southern India: Magmatism and metamorphism in a previously depleted crust. Chemical Geology, 67(1-2):155-163. https://doi.org/10.1016/0009-2541(88)90012-5

Santos E.J., Medeiros V.C. 1999. Constraints from granitic plutonism on Proterozoic crustal growth of the Transverse Zone, Borborema Province, NE Brazil. Brazilian Journal of Geology, 29(1):73-84. https://doi. org/10.25249/0375-7536.1999297384

Sawyer E.W. 2000. Grain-scale and outcrop-scale distribution and movement of melt in a crystallising granite. Transactions of the Royal Society of Edinburgh: Earth Sciences, 91(1-2):73-85. https://doi.org/10.1017/ S0263593300007306

Sawyer E.W. 2008. Atlas of Migmatites. Ottawa: NRC Research Press, 371 p. The Canadian Mineralogist, Special Publication 9.
Scaillet B., Holtz F., Pichavant M. 1998. Phase equilibrium constraints on the viscosity of silicic magmas: 1. Volcanic-plutonic comparison. Journal of Geophysical Research, 103(B11):27257-27266. https://doi. org/10.1029/98JB02469

Schmidt M.W. 1992. Amphibole composition in tonalite as a function of pressure: an experimental calibration of the Al-in-hornblende barometer. Contributions to Mineralogy and Petrology, 110:304-310. https://doi. org/10.1007/BF00310745

Schmidt M.W., Poli S. 2004. Magmatic Epidote. Reviews in Mineralogy and Geochemistry, 56(1):399-430. https://doi.org/10.2138/gsrmg.56.1.399

Schmidt M.W., Thompson A.B. 1996. Epidote in calc-alkaline magmas: An experimental study of stability, phase relationships, and the role of epidote in magmatic evolution. American Mineralogist, 81(3-4):462-474. https:// doi.org/10.2138/am-1996-3-420

Shabani A.A.T., Lalonde A.E., Whalen J.B. 2003. Composition of biotite from granitic rocks of the Canadian Appalachian Orogen: a potential tectonomagmatic indicator? The Canadian Mineralogist, 41(6):1381-1396. https://doi.org/10.2113/gscanmin.41.6.1381

Sial A.N. 1986. Granite-types in northeast Brazil: current knowledge. Brazilian Journal of Geology, 16(1):54-72. https://doi. org/10.25249/0375-7536.19865472

Sial A.N. 1990. Epidote-bearing calc-alkalic granitoids in Northeast Brazil. Brazilian Journal of Geology, 20(1-4):88-100. https://doi. org/10.25249/0375-7536.199088100

Sial A.N. 1993. Contrasting metaluminous magmatic epidote-bearing granitic suites from two Precambrian Foldbelts in Northeast Brazil. Anais da Academia Brasileira de Ciências, 65(Suppl.):141-162.

Sial A.N., Ferreira V.P. 1990. Granitoids in northeastern Brazil: Oxygen and sulfur isotope compositions and depths of emplacement. Journal of South American Earth Sciences, 3(2-3):103-112. https://doi. org/10.1016/0895-9811(90)90023-T

Sial A.N., Ferreira V.P. 2015. Magma associations in Ediacaran granitoids of the Cachoeirinha-Salgueiro and Alto Pajeú terranes, northeastern Brazil Forty years of studies. Journal of South American Earth Sciences, 68:113-133. https://doi.org/10.1016/j.jsames.2015.10.005

Sial A.N., Ferreira V.P., Fallick A.E., Cruz M.J.M. 1998. Amphibole-rich clots in calc-alkalic granitoids in the Borborema province, northeastern Brazil. Journal of South American Earth Sciences, 11 (5):457-471. https://doi. org/10.1016/S0895-9811(98)00034-0

Sial A.N., Ferreira V.P., Santos E.J. 1997. Magmatic epidote-bearing granitoids and ultrapotassic magmatism of the Borborema Province, northeast Brazil. In: International Symposium on Granites and Associated Mineralizations, 2., 1997. Excursions Guide. Salvador. p. 35-54.

Sial A.N., Toselli A.J., Saavedra J., Parada M.A., Ferreira V.P. 1999. Emplacement, petrological and magnetic susceptibility characteristics of diverse magmatic epidote-bearing granitoid rocks in Brazil, Argentina and Chile. Lithos, 46(3):367-392. https://doi.org/10.1016/ S0024-4937(98)00074-7

Sial A.N., Vasconcelos P.M., Ferreira V.P., Pessoa R.R., Brasilino R.G., Morais Neto J.M. 2008. Geochronological and mineralogical constraints on depth of emplacement and ascension rates of epidote-bearing magmas from northeastern Brazil. Lithos, 105(3-4):225-238. https://doi.org/10.1016/j. lithos.2008.04.002

Silva T.R. 2017. Granitos da porção sudeste do batólito águas Belas-Canindé, domínio Pernambuco-Alagoas: Geoquímica, petrologia, geocronologia e caracterização geotectônica. PhD thesis, Universidade Federal de Pernambuco, Recife, 190 p. https://doi.org/10.13140/RG.2.2.16693.70888

Silva T.R., Ferreira V.P., Lima M.M.C., Sial A.N. 2016. Two stage mantlederived granitic rocks and the onset of the Brasiliano orogeny: Evidence from $\mathrm{Sr}$, Nd, and O isotopes. Lithos, 264:189-200. https://doi.org/10.1016/j. lithos.2016.08.030

Silva T.R., Ferreira V.P., Lima M.M.C., Sial A.N. 2019. Geochemical and isotope evidence for mantle-derived source rock of high- $\mathrm{K}$ calcalkaline I-type granites, Pernambuco-Alagoas Domain, northeastern Brazil. International Journal of Earth Sciences, 108:1095-1120. https://doi. org/10.1007/s00531-019-01696-9 
Silva T.R., Ferreira V.P., Lima M.M.C., Sial A.N., Silva J.M.R. 2015. Synkinematic emplacement of the magmatic epidote bearing Major Isidoro tonalite-granite batholith: Relicts of an Ediacaran continental arc in the Pernambuco-Alagoas domain, Borborema Province, NE Brazil. Journal of South American Earth Sciences, 64(Part I):1-13. https://doi.org/10.1016/j. jsames.2015.09.002

Silva Filho A.F., Guimarães I.P., Santos L., Armstrong R., Van Schmus W.R. 2016. Geochemistry, U-Pb geochronology, $\mathrm{Sm}-\mathrm{Nd}$ and O isotopes of ca. 50 Ma long Ediacaran High-K Syn-Collisional Magmatism in the Pernambuco Alagoas Domain, Borborema Province, NE Brazil. Journal of South American Earth Sciences, 68:134-154. https://doi.org/10.1016/j.jsames.2015.12.013

Silva Filho A.F., Guimarães I.P., Van Schmus W.R. 2002. Crustal Evolution of the Pernambuco-Alagoas Complex, Borborema Province, NE Brazil: Nd Isotopic Data from Neoproterozoic Granitoids. Gondwana Research, 5(2):409-422. https://doi.org/10.1016/S1342-937X(05)70732-2

Silva Filho A.F., Guimarães I.P., Van Schmus W.R., Armstrong R., Silva J.M.R., Osako L.S., Cocentino L.M. 2014. SHRIMP U-Pb zircon geochronology and $\mathrm{Nd}$ signatures of supracrustal sequences and orthogneisses constrain the Neoproterozoic evolution of the Pernambuco-Alagoas domain, southern part of Borborema Province, NE Brazil. International Journal of Earth Sciences, 103:2155-2190. https://doi.org/10.1007/s00531-014-1035-4

Solano J.M.S., Jackson M.D., Sparks R.S.J., Blundy J.D., Annen C. 2012. Melt segregation in deep crustal hot zones: a mechanism for chemical differentiation, crustal assimilation and the formation of evolved magmas. Journal of Petrology, 53(10):1999-2026. https://doi.org/10.1093/ petrology/egs041

Tchameni R., Sun F., Dawaï D., Danra G., Tékoum L., Nomo Negue E., Vanderhaeghe O., Nzolang C., Dagwaï N. 2016. Zircon dating and mineralogy of the Mokong Pan-African magmatic epidote-bearing granite (North Cameroon). International Journal of Earth Sciences, 105:1811-1830. https://doi.org/10.1007/s00531-015-1276-x

Terentiev R.V., Savko K.A., Santosh M., Korish E.H., Sarkisyan L.S. 2016. Paleoproterozoic granitoids of the Losevo terrane, East European Craton: Age, magma source and tectonic implications. Precambrian Research, 287:48-72. http://dx.doi.org/10.1016/j.precamres.2016.10.015

Tollari N., Toplis M.J., Barnes S.J. 2006. Predicting phosphate saturation in silicate magmas: An experimental study of the effects of melt composition and temperature. Geochimica et Cosmochimica Acta, 70(6):1518-1536. https://doi.org/10.1016/j.gca.2005.11.024
Tulloch A.J. 1979. Secondary Ca-Al Silicates as Low-Grade Alteration Products of Granitoid Biotite. Contributions to Mineralogy and Petrology, 69:105-117. https://doi.org/10.1007/BF00371854

Vanderhaeghe O. 1999. Pervasive melt migration from migmatites to leucogranite in the Shuswap metamorphic core complex, Canada: control of regional deformation. Tectonophysics, 312(1):35-55. https://doi. org/10.1016/S0040-1951(99)00171-7

Vanderhaeghe O. 2001. Melt segregation, pervasive melt migration and magma mobility: the structural record from pores to orogens. Physics and Chemistry of the Earth, Part A: Solid Earth and Geodesy, 26(4-5):213-223. https://doi.org/10.1016/S1464-1895(01)00048-5

Vanderhaeghe O. 2009. Migmatites, granites and orogeny: Flow modes of partially molten rocks and magmas associated with melt/solid segregation in orogenic belts. Tectonophysics, 477(3-4):119-134. https://doi. org/10.1016/j.tecto.2009.06.021

Van Schmus W.R., Oliveira E.P., Silva Filho A.F., Toteu S.F., Penaye J., Guimarães I.P. 2008. Proterozoic links between the Borborema Province, NE Brazil and the Central African Fold Belt. Geological Society, London, Special Publications, 294(1):69-99. https://doi.org/10.1144/SP294.5

Vyhnal C.R., McSween H.Y., Speer J.A. 1991. Hornblende chemistry in southern Appalachian granitoids: Implication for aluminum hornblende thermobarometry and magmatic epidote stability. American Mineralogist, 76(1-2):176-188.

Watson E.B., Harrison T.M. 1983. Zircon saturation revisited: temperature and composition effects in a variety of crustal magma types. Earth and Planetary Science Letters, 64(2):295-304. https://doi. org/10.1016/0012-821X(83)90211-X

Whitney D.L., Evans B.W. 2010. Abbreviations for names of rock-forming minerals. American Mineralogist, 95(1):185-187. https://doi.org/10.2138/ am.2010.3371

Wones D.R. 1989. Significance of the assemblage titanite + magnetite + quartz in granitic rocks. American Mineralogist, 74(7-8):744-749.

Wones D.R., Eugster H.P. 1965. Stability of biotite: experiment, theory and application. American Mineralogist, 50:1228-1272.

Zen E.-A., Hammarstrom J.M. 1984. Magmatic epidote and its petrologic significance. Geology, 12(9):515-518. https://doi. org/10.1130/0091-7613(1984)12<515:MEAIPS>2.0.CO;2 